MANCHESTER

1824

:这

Economics

Discussion Paper Series

EDP-1307

\title{
Equilibrium Theory under Ambiguity
}

\author{
Wei He \\ Nicholas C. Yannelis
}

April 2013

Economics

School of Social Sciences

The University of Manchester

Manchester M13 9PL 


\title{
Equilibrium Theory under Ambiguity
}

\author{
Wei He* and Nicholas C. Yannelis ${ }^{\dagger}$
}

March 29, 2013

\begin{abstract}
We extend the classical results on the Walras-core existence and equivalence to an ambiguous asymmetric information economies, i.e., economies where agents maximize Maximin Expected Utility (MEU). The interest of considering ambiguity arises from the fact that, in the presence of MEU decision making, there is no conflict between efficiency and incentive compatibility, (contrary to the Bayesian decision making). Our modeling of an ambiguous asymmetric information economy allows for an infinite number of states of the world, and our method of proofs for the existence and equivalence results is different from the Banach space methods used in the literature.
\end{abstract}

JEL classification: D51, D81, D82.

Keywords: Maximin expected utility; Maximin expectations equilibrium; Maximin core; Core-Walras equivalence; Maximin coalitional incentive compatibility

*Department of Mathematics, National University of Singapore, 10 Lower Kent Ridge Road, Singapore 119076. Email: wei.he@nus.edu.sg.

${ }^{\dagger}$ Department of Economics, Henry B. Tippie College of Business, The University of Iowa, 108 John Pappajohn Business Building, Iowa City, IA 52242-1994;

Economics - School of Social Sciences, The University of Manchester, Oxford Road, Manchester M13 9PL, UK. Email: nicholasyannelis@gmail.com. 


\section{Contents}

1 Introduction 3

2 Ambiguous Asymmetric Information Economy $\quad 7$

3 Maximin Expectations Equilibrium and Maximin Core 11

3.1 Basic Definitions . . . . . . . . . . . . . . . . . . . 11

3.2 Existence of MEE and MC . . . . . . . . . . . . . . 13

4 A Continuum Approach $\quad 14$

4.1 Basics . . . . . . . . . . . . . . . . . . . . . 14

4.2 A Continuum Interpretation of the Finite Economy . . . . . . . . 14

4.3 Core Equivalence with a Countable Number of States . . . . . . . 15

4.4 An Extension of Vind's Theorem . . . . . . . . . . . . . . 16

5 Equivalence Theorems $\quad 16$

6 Efficiency and Incentive Compatibility under Ambiguity 17

6.1 Efficiency and Incentive Compatibility . . . . . . . . . . . . . 17

6.2 Maximin Incentive Compatibility . . . . . . . . . . . . . . 18

7 Concluding Remarks $\quad 20$

8 Appendix 21

8.1 Truncated Economy . . . . . . . . . . . . . . . . . 21

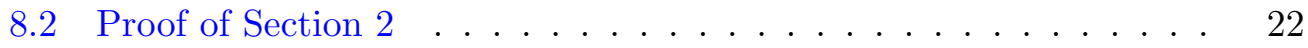

8.3 Proof of Section $3 \ldots \ldots \ldots \ldots \ldots$

8.4 Proof of Section $4 \ldots \ldots \ldots \ldots \ldots$

8.4.1 Proof of Subsection $4.3 \ldots \ldots \ldots \ldots$

8.4 .2 Proof of Subsection $4.4 \ldots \ldots \ldots \ldots$

8.5 Proof of Section $6 \ldots \ldots \ldots \ldots$ 


\section{Introduction}

Modeling the market with uncertainty is of important academic significance and realistic value in economics as most decision making is made under uncertainty. Towards this direction, the Arrow-Debreu 'state contingent model' allows the state of nature of the world to be involved in the initial endowments and payoff functions, which is an enhancement of the deterministic general equilibrium model of ArrowDebreu-McKenzie. According to Arrow-Debreu, agents make contacts ex ante (in period one) before the state of nature is realized and once the state is realized (in period two) the contract is executed and consumption takes place. The issue of incentive compatibility doesn't arise in this model, as all the information is symmetric. However, for the state contingent model to make sense one must assume that there is an exogenous court or government that enforces the contract ex post, otherwise agents may find it beneficial to renege. Radner $(1968,1982)$ extended the analysis of Arrow and Debreu by introducing asymmetric (differential) information; in particular, each agent is now characterized by his own private information, a random initial endowment, a random utility function and a prior. The private information is modeled as a partition of a finite state space and the allocation of each agent is assumed to be measurable with respect to his own private information. This means each agent only knows the atom of his partition including the true state, but cannot distinguish those states within the same atom when making decisions. The Walrasian equilibrium notion in this model is called 'Walrasian expectations equilibrium', or WEE in short. Along this line, Yannelis (1991) proposed a core concept, which is called private core. ${ }^{1}$

The Walrasian expectations equilibrium and private core share some interesting properties (in fact, the Walrasian expectations equilibrium is a strict subset of the private core): without the assumption of free disposal, whenever agents are Bayesian expected utility maximizers and allocations are private information measurable, the two above notions are both Bayesian incentive compatible and private information measurable efficient (see Koutsougeras and Yannelis (1993) and Krasa and Yannelis (1994)). However, these solution concepts are only efficient in the second best sense, i.e., they are only private information measurable efficient allocations and may result in a possible welfare loss (recall that from Holmstrom and Myerson (1983) we know that with the standard/Bayesian expected utility it is not possible to have allocations which are both first best efficient and also incentive compatible). The existence of WEE in a free disposal economy can be found in Radner $(1968,1982)$. However, the free disposal WEE allocations may

\footnotetext{
${ }^{1}$ For a recent treatment of general equilibrium with asymmetric information see the books Glycopantis and Yannelis (2005) and Marakulin (2013).
} 
be not incentive compatible (see Glycopantis et al. (2003)). Furthermore, if we require non-free disposal, then a WEE may not exist with positive price (see Einy and Shitovitz (2001)). Therefore, a natural question arises:

Can one find an appropriate framework in the asymmetric information economy such that the existence of equilibrium and core notions continues to hold and furthermore, these notions are both incentive compatible and first best efficient?

A crucial assumption in the frameworks of Radner $(1968,1982)$ and Yannelis (1991) is that agents maximize Bayesian expected utilities. Nevertheless, from Ellsberg (1961), there is a huge literature which criticizes the Bayesian paradig$\mathrm{m}$ and explores the non-expected utility theory. The maximin expected utility of Gilboa and Schmeidler (1989) is one of the successful alternatives. Indeed, recently de Castro et al. (2011) and de Castro and Yannelis (2010) applied the maximin expected utility to an asymmetric information economy with with a finite number of states of nature, ${ }^{2}$ and introduced various core and Walrasian equilibrium notions. With the maximin expected utilities, agents take into account the worst possible state that can occur and choose the best possible allocations. de Castro et al. (2011) proved that the ex ante equilibrium and core notions based on the maximin expected utility, which are called maximin expectations equilibrium (MEE) and maximin core (MC) therein, are incentive compatible in the economy without free disposal. Moreover, it is noteworthy that since the allocations are not required to be measurable with respect to agents' private information, MEE and MC allocations are also first best efficient. Therefore, the conflict between efficiency and incentive compatibility is solved in this new approach. Indeed de Castro and Yannelis (2010) showed that the conflict of incentive compatibility and first best efficiency is inherent in the standard expected utility decision making (Bayesian) and it is resolved only when agents maximize the maximin expected utility (MEU). In particular, they proved that the MEU is a necessary condition for efficient allocations to be incentive compatible. The above work has left a main open question:

Can one obtain the classical core-Walras existence and equivalence results for asymmetric information economies where agents are ambiguous (i.e., are MEU maximizers) and also the state space is not necessarily finite?

\footnotetext{
${ }^{2} \mathrm{MEU}$ is first applied to a general equilibrium model of an asymmetric information economy by Correia-da-Silva and Hervés-Beloso (2009). They proved the existence of the ex ante Walrasian equilibrium in an asymmetric information economy with maximin preferences and a finite state space. However, their setup is different from ours and they do not consider the issue of incentive compatibility. For other applications of ambiguity in general equilibrium model, see Araujo et al. (2011).
} 
An affirmative answer to this question is of great importance because not only this way one develops a new equilibrium theory where there is no conflict between efficiency and incentive compatibility, but also such positive results could become the main tool for applications in other fields of Economics.

The first aim of this paper is to prove the existence of the maximin expectations equilibrium and maximin core in a non-free disposal economy with countably many states of nature. Since there is a countable number of states in the economy, the allocations are infinite dimensional. Unlike the proofs of the existence of Walrasian expectations equilibrium in an economy with an infinite dimensional commodity space (e.g. Bewley (1972) and Podczeck and Yannelis (2008)), our proof does not use Banach space methods; as a matter of fact, the space of allocations in this paper may not be a Banach space. Specifically, we proceed in three steps: first, we prove the existence of MEE in the economy with finitely many states; second, we generate a sequence of economies with finitely many states which are all truncations of the economy with countably many states; third, we show that the sequence of equilibria generated by the sequence of finite state economies has a convergent point, which is the maximin expectations equilibrium in the economy with a countable number of states. As a corollary we obtain that the consistency between incentive compatibility and efficiency also holds with a countable number of states.

The second aim of the current paper is to prove a core equivalence theorem for an economy with asymmetric information where agents are ambiguous (i.e., maximize MEU). In a finite agent framework and complete information, Debreu and Scarf (1963) considered a sequence of replicated economy and showed that the set of non-blocked allocations in every replicated economy converges to the set of Walrasian equilibria. In Section 4, we follow the Debreu-Scarf approach and establish a similar equivalence result for an equal treatment economy with asymmetric information, a countable number of states and MEU preferences. In an atomless economy with complete information, Schmeidler (1972), Grodal (1972) and Vind (1972) improved the core-Walras equivalence theorem of Aumann (1964), by showing that if an allocation is not in the core, then it can be blocked by a non-negligible coalition with any given measure less than 1. Hervés-Beloso et al. (2005a,b) first extended this result to a asymmetric information economy with the equal treatment property and with an infinite dimensional commodity space by appealing to the finite dimensional Lyapunov's theorem. Based on an infinite dimensional version of Lyapunov's theorem, Bhowmik and Cao (2012a) established a similar result in an atomless economy whose commodity space is an ordered Banach space having an interior point in its positive cone, and Bhowmik and Cao (2012b) obtained extensions in an asymmetric information economy with the equal treatment prop- 
erty whose commodity space is a Banach lattice. All the above results rely on the Bayesian expected utility formulation and therefore the conflict of efficiency and incentive compatibility still holds despite the non atomic measure space of agents. ${ }^{3}$ Our Theorem 4 is an extension of Vind's theorem to the asymmetric information economy with the equal treatment property and a countable number of states of nature, where agents behave as maximin expected utility maximizers. Thus, our new core equivalence theorem for the MEU framework, resolves the inconsistency of efficiency and incentive compatibility.

Finally, we provide two characterizations for maximin expectations equilibrium. In the complete information economy with finite agents, Aubin (1979) introduced a new approach that at a first glance seems to be different from the Debreu-Scarf; however one can show that they are essentially equivalent. Aubin considered a veto mechanism in the economy when a coalition is formed; in particular, agents are allowed to participate with any proportion of their endowments. The core notions defined by the veto mechanism, is called Aubin core and it coincides with the Walrasian equilibrium allocations. The approach of Aubin has been extended to an asymmetric information economy to characterize the Walrasian expectations equilibrium (see for example Graziano and Meo (2005), Hervés-Beloso et al. (2005b) and Bhowmik and Cao (2012b)). Another approach to characterize the Walrasian expectations equilibrium is due to Hervés-Beloso et al. (2005a,b). They showed that the Walrasian expectations equilibrium allocation cannot be privately blocked by the grand coalition in any economy with the initial endowment redistributed along the direction of the allocation itself. This approach has been extended to a pure exchange economy with an atomless measure space of agents and finitely many commodities, and an asymmetric information economy with an infinite dimensional commodity space (e.g., see Hervés-Beloso and E. Moreno-García (2008), Bhowmik and Cao (2012b) and Bhowmik and Cao (2013)). Our Theorem 5 and 6 extended these two characterizations to the asymmetric information economy with ambiguous agents and with countably many states of nature.

The paper is organized as follows. Section 2 states the model of ambiguous asymmetric information economies with a countable number of states and discusses main assumptions. Section 3 introduces the maximin expectations equilibrium and maximin core and proves their existences. Section 4 extends the maximin expectations equilibrium and maximin core to an economy with a continuum of agents, and interprets the asymmetric information economy with finite agents as a continuum economy with finite types. In addition, two core-Walras equivalence theorems and an extension of Vind's result are given for an asymmetric information

\footnotetext{
${ }^{3}$ As the work of Sun and Yannelis (2008) indicates, even with an atomless measure space of agents we cannot guarantee that WEE allocations are incentive compatible
} 
economy with a countable number of states. Section 5 contains two different characterizations of maximin expectations equilibrium by using the maximin blocking power of the grand coalition. Section 6 shows maximin efficient allocations are coalitional incentive compatible in economies with finite agents and atomless economies with the equal treatment property. Section 7 collects some concluding remarks and open questions. The appendix (Section 8) discusses the truncated economy for an ambiguous asymmetric information economy with a countable number of states and contains longer proofs.

\section{Ambiguous Asymmetric Information Econ- omy}

We introduce a model of the exchange economy with uncertainty and asymmetric information. The uncertainty is represented by a measurable space $(\Omega, \mathcal{F})$, where $\Omega=\left\{\omega_{n}\right\}_{n \in \mathbb{N}}$ is a countable set and $\mathcal{F}$ is the power set of $\Omega$. $\mathbb{R}_{+}^{l}$ is the commodity space and $I=\{1,2, \cdots, s\}$ is the set of agents.

For each $i \in I, \mathcal{F}_{i}$, the $\sigma$-algebra on $\Omega$ generated by the partition $\Pi_{i}$ of agent $i$, represents the private information. $\Pi_{i}(\omega)$ is the element in the partition $\Pi_{i}$ which includes $\omega$. Therefore, if any state $\omega \in \Omega$ is realized in the interim, agent $i$ only observes the event $\Pi_{i}(\omega) . \pi_{i}$ is $i$ 's private prior defined on $\mathcal{F}_{i}$, i.e., $\pi_{i}$ is a mapping from $\mathcal{F}_{i}$ to $\mathbb{R}_{+}$such that $\sum_{E \in \Pi_{i}} \pi_{i}(E)=1$ and $\pi_{i}(E)>0$ for every $E \in \Pi_{i} . \pi_{i}$ can be incomplete, i.e., the probability of each element in the information partition $\Pi_{i}$ is well defined, but not the probability of the event $\{\omega\}$ for every $\omega \in \Omega{ }^{4} u_{i}\left(\omega, x_{i}\right)$ is the positive ex post utility function of agent $i$ at state $\omega$ with consumption plan $x_{i} . e_{i}: \Omega \rightarrow \mathbb{R}_{+}^{l}$ is $i$ 's random initial endowment.

$\mathcal{E}$ denotes the ambiguous asymmetric information economy, where

$$
\mathcal{E}=\left\{(\Omega, \mathcal{F}) ;\left(\mathcal{F}_{i}, u_{i}, e_{i}, \pi_{i}\right): i \in I=\{1, \ldots, s\}\right\}
$$

A price vector $p$ is a nonzero positive ${ }^{5}$ function from $\Omega$ to the simplex of $\mathbb{R}_{+}^{l}$. Without loss of generality, we may assume that $\triangle$ denotes the set of all price vectors.

$$
\triangle=\left\{p \in\left(\mathbb{R}_{+}^{l}\right)^{\infty}: \sum_{\omega \in \Omega} \sum_{j=1}^{l} p(\omega, j)=1\right\},
$$

where $p(\omega, j)$ is the price of the commodity $j$ at state $\omega$.

\footnotetext{
${ }^{4}$ This setup is consistent with the MEU assumption, but obviously inconsistent with the Bayesian expected utility where all agents are assumed to know the probability of every state of nature.

${ }^{5} p(\omega)$ is a nonzero function means that $p$ is not a 0 constant function, but it is possible that $p(\omega)=0$ for some $\omega$.
} 
There are three stages in this economy: at the ex ante stage $(t=0)$, the information partition, the economy structure and the price are all common knowledge; at the interim stage $(\mathrm{t}=1)$, each individual $i$ learns his private information $\Pi_{i}(\omega)$ which includes the true state $\omega$, and makes his consumption plan; at the ex post stage $(\mathrm{t}=2)$, agent $i$ receives the endowment and consumes according to his plan.

An allocation is a mapping $x$ from $I \times \Omega$ to $\mathbb{R}_{+}^{l}$. For all $i \in I, L_{i}=\left\{x_{i}\right.$ : $x_{i}(\omega) \in \mathbb{R}_{+}^{l}$ for all $\left.\omega \in \Omega\right\}$ is the set of all random allocations and $e_{i} \in L_{i}$. It is easy to see that $L_{i}$ is $\left(\mathbb{R}_{+}^{l}\right)^{\infty}$ for every $i$ and $\triangle$ is a subset of $\left(\mathbb{R}_{+}^{l}\right)^{\infty}$. If $x_{i} \in L_{i}$ and $p \in \triangle$, we denote $\sum_{\omega \in \Omega} p(\omega) \cdot x_{i}(\omega)$ as $p \cdot x_{i}$, which could be infinity.

Suppose $x$ is an allocation, for any $i \in I, x_{i}(\omega)$ is a vector in $\mathbb{R}_{+}^{l}$, it represents the allocation at the state $\omega$, and $x_{i}(\omega, j)$ denotes the allocation of commodity $j$ at the state $\omega$. Suppose $\|\cdot\|$ is the natural norm in $\mathbb{R}_{+}^{l}, x_{i}$ and $y_{i}$ are two points in $\left(\mathbb{R}_{+}^{l}\right)^{\infty}$, then the metric

$$
d\left(x_{i}, y_{i}\right)=\sup _{n \in \mathbb{N}}\left[\frac{1}{n} \min \left\{\left\|x_{i}\left(\omega_{n}\right)-y_{i}\left(\omega_{n}\right)\right\|, 1\right\}\right]
$$

induces the product topology on $\left(\mathbb{R}_{+}^{l}\right)^{\infty}$. Hereafter all statements on $\left(\mathbb{R}_{+}^{l}\right)^{\infty}$ about continuity, convergence, etc., will be with respect to this topology.

An allocation $x$ is said to be feasible if $\sum_{i \in I} x_{i}=\sum_{i \in I} e_{i}$, i.e., $\forall \omega \in \Omega$,

$$
\sum_{i \in I} x_{i}(\omega)=\sum_{i \in I} e_{i}(\omega)
$$

The feasibility here indicates that the economy is non-free disposal.

Assumption (E). 1. For each $i \in I, e_{i}$ is $\mathcal{F}_{i}$-measurable. ${ }^{6}$

2. $e_{i} \gg 0$ for any $i \in I$.

3. $\exists M>0, \forall \omega \in \Omega$ and $1 \leq j \leq l, \sum_{i \in I} e_{i}(\omega, j) \leq M$.

Assumption (E) is about the endowment. Condition (1) says that each agent's endowment should be measurable with respect to his private information, otherwise the agent may disclose the true state from his endowment. Condition (2) says for every agent $i, e_{i}$ is an interior point of $\left(\mathbb{R}_{+}^{l}\right)^{\infty}$. Condition (3) says the resource of the economy is limited no matter what the state is, this condition will be automatically satisfied if there are only finitely many states.

Assumption (U). 1. For each $\omega \in \Omega$ and $i \in I, u_{i}(\omega, \cdot)$ is continuous, strictly increasing and concave.

2. For each $i \in I$ and $x \in \mathbb{R}_{+}^{l}, u_{i}(\cdot, x)$ is $\mathcal{F}_{i}$-measurable. ${ }^{7}$

${ }^{6}$ Clearly, if $e_{i}$ is independent of $\omega$, then it is $\mathcal{F}_{i}$-measurable.

${ }^{7}$ If $u_{i}$ is state independent, then it is automatically $\mathcal{F}_{i}$-measurable. 
3. $\forall a \in \mathbb{R}_{+}^{l}$, if there exists $K_{0}>0$, such that $|a(j)| \leq K_{0}$ for $1 \leq j \leq l$, then $\exists K>0$, such that $0 \leq u_{i}(\omega, a) \leq K, \forall i \in I$ and $\forall \omega \in \Omega$. $u_{i}(\omega, 0)=0$ for all $i \in I$ and $\omega \in \Omega$.

Assumption (U) is about the utility. Condition (1) and (2) are standard in the literature. Condition (3) basically says that there is no 'bubble' in the world, i.e., people's utility cannot be arbitrarily large with limited goods. This condition can be removed if $\Omega$ is finite: for each $i \in I$ and every $\omega, u_{i}(\omega, a)$ is continuous at $a$, if $a$ is bounded, then $u_{i}(\omega, \cdot)$ is bounded, since there are only finitely many states, $u_{i}(\omega, \cdot)$ is uniformly bounded among all $\omega$. Moreover, $u_{i}(\omega, 0)=0$ is a normalization, meaning that people have no payoff if they have no goods.

For every agent $i$, his private prior may be incomplete and the allocation in $L_{i}$ is not required to be $\mathcal{F}_{i}$-measurable, thus agents cannot evaluate the allocation based on the Bayesian expected utility. In the current paper, we will adopt the maximin preference axiomatized by Gilboa and Schmeidler (1989). Let $\mathcal{M}_{i}$ be the set of probability measures on $\mathcal{F}$ which agree with $\pi_{i}$ on $\mathcal{F}_{i}$, i.e.,

$$
\mathcal{M}_{i}=\left\{\mu: \mathcal{F} \rightarrow[0,1]: \mu(E)=\pi_{i}(E), \forall E \in \mathcal{F}_{i}\right\}
$$

Then for any two allocation $x_{i}, y_{i} \in L_{i}$, agent $i$ prefers the allocation $x_{i}$ to the allocation $y_{i}$ if

$$
\inf _{\mu \in \mathcal{M}_{i}} \sum_{\omega \in \Omega} u_{i}\left(\omega, x_{i}(\omega)\right) \mu(\omega) \geq \inf _{\mu \in \mathcal{M}_{i}} \sum_{\omega \in \Omega} u_{i}\left(\omega, y_{i}(\omega)\right) \mu(\omega)
$$

de Castro and Yannelis (2010) shows that the inequality above is equivalent to the following one: ${ }^{8}$

$$
\sum_{E_{i} \in \Pi_{i}}\left[\inf _{\omega \in E_{i}} u_{i}\left(\omega, x_{i}(\omega)\right)\right] \pi_{i}\left(E_{i}\right) \geq \sum_{E_{i} \in \Pi_{i}}\left[\inf _{\omega \in E_{i}} u_{i}\left(\omega, y_{i}(\omega)\right)\right] \pi_{i}\left(E_{i}\right)
$$

In this paper, we will follow the second formulation. For any allocation $\left\{x_{i}\right\}_{i \in I}$, the maximin ex ante utility of agent $i$ is:

$$
V_{i}\left(x_{i}\right)=\sum_{E_{i} \in \Pi_{i}}\left[\inf _{\omega \in E_{i}} u_{i}\left(\omega, x_{i}(\omega)\right)\right] \pi_{i}\left(E_{i}\right)
$$

Let $\mathcal{M}_{i}(\omega)$ be the set of all probabilities with support concentrated at $\Pi_{i}(\omega)$, then

${ }^{8}$ First, we use 'inf' in these two inequalities instead of 'min' used in de Castro and Yannelis (2010), since there are infinite states here. The existence of infimum is guaranteed since the ex post utility function is nonnegative. Thus the ex ante utility $V_{i}$ is well defined.

Second, although de Castro and Yannelis (2010) only argued these two inequalities are equivalent when there are finitely many states, this observation is still true in our context. 
the maximin interim utility of agent $i$ with allocation $x_{i}$ at state $\omega$ is

$$
v_{i}\left(\omega, x_{i}\right)=\inf _{\mu \in \mathcal{M}_{i}(\omega)} E_{\mu}\left[u_{i}\left(\cdot, x_{i}(\cdot)\right)\right]=\inf _{\omega_{1} \in \Pi_{i}(\omega)} u_{i}\left(\omega_{1}, x_{i}\left(\omega_{1}\right)\right) .
$$

We will slightly abuse the notations by letting $v_{i}\left(E_{i}, x_{i}\right)=\inf _{\omega \in E_{i}} u_{i}\left(\omega, x_{i}(\omega)\right)$, where $E_{i}$ is an atom in the information partition $\Pi_{i}$ of agent $i$.

The last assumption (I) is used to guarantee the continuity of the maximin ex ante utility.

Assumption (I). ${ }^{9}$ There exists an increasing sequence of subsets $\left\{F_{n}\right\}_{n \in \mathbb{N}}$ such that

1. $\forall n, F_{n} \subseteq \Omega$ is finite and $\cup_{n \in \mathbb{N}} F_{n}=\Omega$;

2. For any agent $i, \forall E_{i} \in \Pi_{i}, \forall n \in \mathbb{N}, E_{i} \cap F_{n}=\emptyset$ or $E_{i} \subseteq F_{n}$.

This assumption will be automatically satisfied in either of the following cases: (1) $\Omega$ is finite, then we can choose $F_{n}=\Omega$ for all $n \in \mathbb{N}$; (2) the information of all agents is symmetric, then we can choose $F_{n}=\left\{\omega_{i}\right\}_{1 \leq i \leq n}$. If this assumption holds, then for any $\omega$, the states which agent $i$ cannot distinguish are at most finite. Thus we have:

$$
V_{i}\left(x_{i}\right)=\sum_{E_{i} \in \Pi_{i}}\left[\min _{\omega \in E_{i}} u_{i}\left(\omega, x_{i}(\omega)\right)\right] \pi_{i}\left(E_{i}\right)
$$

it is similar for the maximin interim utility.

We define the continuity property of the maximin ex ante utility function on the space of allocations.

Definition 1. A function $V$ is said to be uniformly continuous on $\left(\mathbb{R}_{+}^{l}\right)^{\infty}$ if for all $x^{n}$ and $z$ in $\left(\mathbb{R}_{+}^{l}\right)^{\infty}, d\left(x^{n}, z\right) \rightarrow 0$ implies $\left|V\left(x^{n}\right)-V(z)\right| \rightarrow 0$.

Proposition 1. If Assumptions (U.3) and (I) hold, $u_{i}(\omega, \cdot)$ is continuous for all $\omega \in \Omega$ and $i \in I$, then $V_{i}$ is uniformly continuous on the set

$$
G=\left\{x \in L_{i}: 0 \leq x(\omega, j) \leq M \text { for all } \omega \in \Omega \text { and } j=1,2, \cdots, l .\right\}
$$

where $M>0$.

Proof. See appendix.

\footnotetext{
${ }^{9}$ This condition basically says that the information partitions of all agents cannot be too asymmetric.
} 


\section{Maximin Expectations Equilibrium and Max- imin Core}

\subsection{Basic Definitions}

In this section we define the notions of the maximin core (MC) and maximin expectations equilibrium (MEE).

Given a price vector $p$, the budget set of agent $i$ is defined as follow:

$$
B_{i}(p)=\left\{x_{i} \in L_{i}: \sum_{\omega \in \Omega} p(\omega) \cdot x_{i}(\omega) \leq \sum_{\omega \in \Omega} p(\omega) \cdot e_{i}(\omega)\right\} .
$$

Definition 2. An allocation $x$ is said to be a maximin expectations equilibri$\boldsymbol{u m}$ allocation for the economy $\mathcal{E}$, if there exists a price vector $p$ such that for any agent $i$

1. $x_{i}$ maximizes $V_{i}(\cdot)$ subject to the budget set $B_{i}(p)$;

2. $x$ is feasible.

The following definition of a core concept in the current context implies that coalitions of agents cannot cooperate to become better off in terms of MEU.

Definition 3. A feasible allocation $x$ is said to be a maximin core allocation for the economy $\mathcal{E}$, if there do not exist a coalition $C \subseteq I, C \neq \emptyset$, and an allocation $\left\{y_{i} \in L_{i}\right\}_{i \in C}$ such that

(i) $V_{i}\left(y_{i}\right)>V_{i}\left(x_{i}\right)$ for all $i \in C$;

(ii) $\sum_{i \in C} y_{i}(\omega)=\sum_{i \in C} e_{i}(\omega)$ for all $\omega \in \Omega$.

The allocation is said to be maximin efficient if $C=I$.

Remark 1. If Bayesian expected utilities, instead of maximin expected utilities, are used in Definition 2 and 3, and the private information measurability assumption is imposed on allocations, then these two solution concepts are Walrasian expectations equilibrium and private core defined in Radner (1968) and Yannelis (1991) respectively.

The following example shows that MEE could exist in an economy where there is no (non-free disposal) WEE, and the efficiency of MEE allocations can be strictly higher than the efficiency of (free disposal) WEE allocations.

Example 1. ${ }^{10}$ Consider the following economy with one commodity, the agent space is $I=\{1,2\}$ and the state space is $\Omega=\{a, b, c\}$. The initial endowments

\footnotetext{
${ }^{10}$ This example has been analyzed in Glycopantis et al. (2003) in Bayesian preference setting for the existence and incentive compatibility of Walrasian expectations equilibrium and private core, and in Liu and Yannelis (2013) in maximin preference setting for the existence and incentive compatibility of maximin core.
} 
and information partitions of agents are given by

$$
\begin{aligned}
& e_{1}=(5,5,0), \Pi_{1}=\{\{a, b\},\{c\}\} ; \\
& e_{2}=(5,0,5), \Pi_{2}=\{\{a, c\},\{b\}\} .
\end{aligned}
$$

It is also assumed that for $i \in I, u_{i}\left(\omega, x_{i}\right)=\sqrt{x_{i}}$, which is strictly concave and monotone in $x_{i}$, and the priors for both agents are the same: $\mu(\{\omega\})=\frac{1}{3}$ for every $\omega \in \Omega$.

Suppose that agents are both Bayesian expected utility maximizers, it can be easily checked that there is no (non-free disposal) WEE with positive prices. If we allow for free disposal, $x_{1}=(4,4,1)$ and $x_{2}=(4,1,4)$ is a (free disposal) WEE allocation with the equilibrium price $p(a)=0$ and $p(b)=p(c)=\frac{1}{2}$. However, this allocation is not incentive compatible (see Example 2 in Section 6 for details).

If agents are maximin expected utility maximizers, then there exists an MEE $(y, p)$, where $y_{1}=(5,4,1), y_{2}=(5,1,4)$ and $p(a)=0, p(b)=p(c)=\frac{1}{2}$. This allocation is maximin incentive compatible (see Example 3 in Section 6).

If state $b$ or $c$ realizes, the ex post utility of agent 1 will be the same in both Bayesian preference setting and maximin preference setting, since $x_{1}(b)=y_{1}(b)$ and $x_{1}(c)=y_{1}(c)$. But if state $a$ occurs, the ex post utility of agent 1 with maximin preference will be strictly higher than that in the Bayesian preference setting, since

$$
x_{1}(a)=4<5=y_{1}(a) .
$$

Therefore, the maximin preference allows agents to get incentive compatible allocations and reach higher efficiency, contrary to the Bayesian preference.

The lemma below shows that the set of maximin expectations equilibrium allocations is included in the set of maximin core allocations.

Lemma 1. The set of MEE allocations is a subset of the $M C$, hence any maximin expectations equilibrium allocation is maximin efficient.

Proof. Let $x$ be an MEE allocation and $p$ be the corresponding equilibrium price vector. Suppose $x \notin M C$, then there is a coalition $C \subseteq I, C \neq \emptyset$, and an allocation $\left\{y_{i} \in L_{i}\right\}_{i \in C}$ satisfying $\sum_{i \in C} y_{i}=\sum_{i \in C} e_{i}, V_{i}\left(y_{i}\right)>V_{i}\left(x_{i}\right)$ for all $i \in C$.

Since $V_{i}\left(y_{i}\right)>V_{i}\left(x_{i}\right)$ for all $i \in C$, by the definition of MEE, we have $y_{i} \notin B_{i}(p)$, thus $p \cdot y_{i}>p \cdot e_{i}$ for all $i \in C$.

Sum up all the inequality and we get

$$
p \cdot \sum_{i \in C} y_{i}>p \cdot \sum_{i \in C} e_{i}
$$


Which contradicts the condition that $\sum_{i \in C} y_{i}=\sum_{i \in C} e_{i}$.

Therefore, $M E E \subseteq M C$.

This inclusion can be strict. It is clear that both the Arrow-Debreu 'state contingent model' and the deterministic general equilibrium model are special cases of our model: if $\mathcal{F}_{i}=\mathcal{F}$ for every $i \in I$ then the maximin expected utility coincides with the Bayesian expected utility and $\mathcal{E}$ is indeed the state contingent model; if $\Omega$ is a singleton, then $\mathcal{E}$ is the deterministic model. Moreover, it is well known that in those two models, the set of core allocations could strictly contain the set of Walrasian equilibrium allocations.

\subsection{Existence of MEE and MC}

We now turn to the issue of the existence of MEE. ${ }^{11}$ The proof will be divided into two parts. In the first part we consider an ambiguous asymmetric information economy where there are only finitely many states. This result is stated as a lemma, the proof is left in the appendix.

Lemma 2. Suppose that Assumptions (E.2), (U.1) hold and $\Omega=\left\{\omega_{1}, \cdots, \omega_{r}\right\}$ is finite. Then there exists an $M E E$.

In the second part we use a sequence of finite states economy to approximate the countable states economy; in particular, the sequence of equilibria for these finite states economies has a convergent point, which is exactly the equilibrium of the countable states economy.

A generalization of Lemma 2 to the countable states case is given below.

Theorem 1. For the ambiguous asymmetric information economy $\mathcal{E}$, if Assumptions (E), (U) and (I) hold, then there exists an MEE. ${ }^{12}$

Proof. See appendix.

Based on Theorem 1 and Lemma 1, it is straightforward to show that the maximin core is also nonempty.

Corollary 1. Under the conditions of Theorem 1, the maximin core is nonempty.

\footnotetext{
${ }^{11}$ Podczeck et al. (2008) showed that the WEE may not exist in an asymmetric information economy with Bayesian expected utility maximizers and infinitely many states. Our assumption (I) does not hold in their model since the requirement that $F_{n}$ is finite for each $n \in \mathbb{N}$ could fail there.

${ }^{12}$ Assumptions (E.1) and (U.2)) are not needed in the proof of this theorem.
} 


\section{A Continuum Approach}

\subsection{Basics}

Now we introduce the maximin expectations equilibrium and maximin core for an atomless economy. The atomless probability space $(T, \mathcal{T}, \lambda)$ denotes the agent space. We can define an atomless ambiguous asymmetric information economy as

$$
\mathcal{E}_{0}=\left\{(\Omega, \mathcal{F}) ;\left(\mathcal{F}_{t}, u_{t}, e_{t}, \pi_{t}\right): t \in T\right\} .
$$

An allocation in the continuum economy $\mathcal{E}_{0}$ is a mapping $f$ from $T \times \Omega$ to $\mathbb{R}_{+}^{l}$ such that $f(\cdot, \omega)$ is integrable for every $\omega \in \Omega$, the allocation is said to be feasible if $\int_{T} f(t, \omega) \mathrm{d} \lambda(t)=\int_{T} e(t, \omega) \mathrm{d} \lambda(t)$ for every $\omega \in \Omega$.

A coalition in $T$ is a set $S \in \mathcal{T}$ such that $\lambda(S)>0$. An allocation $f$ is maximin blocked by a coalition $S$ in the economy $\mathcal{E}_{0}$ if there exists $g: S \times \Omega \rightarrow \mathbb{R}_{+}^{l}$ such that $\int_{S} g(t, \omega) \mathrm{d} \lambda(t)=\int_{S} e(t, \omega) \mathrm{d} \lambda(t)$ for every $\omega \in \Omega$, and $V_{t}(g(t))>V_{t}(f(t))$ for almost every $t \in S$.

Definition 4. An allocation $f$ is said to be the maximin core for the economy $\mathcal{E}_{0}$ if it is not maximin blocked by any coalition.

Definition 5. An allocation $f$ is said to be a maximin expectations equilibrium allocation for the economy $\mathcal{E}_{0}$, if there exists a price vector $p$ such that

1. $f_{t}$ maximizes $V_{t}(\cdot)$ subject to the budget set $B_{t}(p)$ for almost all $t \in T$;

2. $f$ is feasible.

\subsection{A Continuum Interpretation of the Finite Econo- my}

We associate an atomless economy $\mathcal{E}_{c}$ with the discrete economy $\mathcal{E}$, as in GarcíaCutrín and Hervés-Beloso (1993), Hervés-Beloso et al. (2005a,b) and Bhowmik and Cao $(2012 b)$. The space of agents in $\mathcal{E}_{c}$ is the Lebesgue unit interval $(T, \mathcal{T}, \mu)$ such that $T=\cup_{i=1}^{s} T_{i}$, where $T_{i}=\left[\frac{i-1}{s}, \frac{i}{s}\right)$ for $i=1, \cdots, s-1$ and $T_{s}=\left[\frac{s-1}{s}, 1\right]$. For each agent $t \in T_{i}$, set $\mathcal{F}_{t}=\mathcal{F}_{i}, \pi_{t}=\pi_{i}, u_{t}=u_{i}$ and $e_{t}=e_{i}$. Thus the maximin ex ante utility $V_{t}$ of agent $t$ is $V_{i}$. We refer to $T_{i}$ as the set of agents of type $i$, and

$$
\mathcal{E}_{c}=\left\{(\Omega, \mathcal{F}) ;\left(T, \mathcal{F}_{i}, V_{i}, e_{i}, \pi_{i}\right): i \in I=\{1, \cdots, s\}\right\}
$$

is the economy with the equal treatment property. The allocations in $\mathcal{E}$ and $\mathcal{E}_{c}$ are closely related: for any allocation $f$ in $\mathcal{E}_{c}$, there is an corresponding allocation $x$ in $\mathcal{E}$, where $x_{i}(\omega)=\frac{1}{\mu\left(T_{i}\right)} \int_{T_{i}} f(t, \omega) \mathrm{d} \mu(t)$ for all $i \in I$ and $\omega \in \Omega$; 
conversely, an allocation $x$ in $\mathcal{E}$ can be interpreted as an allocation $f$ in $\mathcal{E}_{c}$, where $f(t, \omega)=x_{i}(\omega)$ for all $t \in T_{i}, \omega \in \Omega$ and $i \in I . f$ is said to be a step allocation if $f(\cdot, \omega)$ is a constant function on $T_{i}$ for any $\omega \in \Omega$ and $i \in I$.

Analogously to the theorems in Hervés-Beloso et al. (2005a,b), the next proposition shows that the maximin expectations equilibrium can be considered equivalent in discrete and continuum approaches.

Proposition 2. Suppose Assumption (U.1) holds, then we have the following properties:

- If $(x, p)$ is an MEE for the economy $\mathcal{E}$, then $(f, p)$ is the MEE for the associated continuum economy $\mathcal{E}_{c}$, where $f(t, \omega)=x_{i}(\omega)$ if $t \in T_{i}$.

- If $(f, p)$ is an MEE for the economy $\mathcal{E}_{c}$, then $(x, p)$ is the MEE for the economy $\mathcal{E}$, where $x_{i}(\omega)=\frac{1}{\mu\left(T_{i}\right)} \int_{T_{i}} f(t, \omega) \mathrm{d} \mu$ for any $\omega \in \Omega$.

The proof is straightforward, interested readers may refer to Theorem 3.1 of Hervés-Beloso et al. (2005b).

\subsection{Core Equivalence with a Countable Number of S- tates}

The core-Walras equivalence theorem has been recently extended to a Bayesian asymmetric information economy. Specifically, Einy et al. (2001) showed that the Walrasian expectations equilibrium is equivalent to the private core for atomless economies with a finite number of commodities in a free disposal setting, Angeloni and Martins-da-Rocha (2009) completed the discussion by proposing appropriate conditions which guarantees the core equivalence result in non-free disposal context. Hervés-Beloso et al. (2005a,b) and Bhowmik and Cao (2012b) followed the Debreu- Scarf approach and showed that the set of Walrasian expectations equilibrium allocations coincides with the private core in the asymmetric information economy with the equal treatment property, finitely many states and infinitely many commodities.

However, all these discussions focus on the asymmetric information economy with Bayesian expected utilities and a finite state space. Our aim here is to examine whether this result is still true when agents are ambiguous (have maximin expected utilities) and the state space is countable. The theorems below show that the core equivalence theorem holds with either of the following conditions:

1. Maximin expected utility and finitely many states;

2. Maximin expected utility, countably many states and the equal treatment property holds. 
Theorem 2. Let $\Omega$ be finite in the atomless economy $\mathcal{E}_{0}$. Assume that (E.2) and (U.1) hold. Then the set of MC allocations coincides with the set of MEE allocations.

We omit the proof since it is standard, interested readers may check that the proof of the core equivalence theorem in Hildenbrand (1974) with minor modifications still holds.

Theorem 3. Suppose Assumptions (E), (U) and (I) hold. ${ }^{13}$ Let the step allocation $f$ be feasible in the associated continuum economy $\mathcal{E}_{c}$. Then $f$ is an MEE allocation if and only if $f$ is an $M C$ allocation.

Proof. See appendix.

\subsection{An Extension of Vind's Theorem}

Hervés-Beloso et al. (2005a,b) and Bhowmik and Cao (2012b) extended Vind's theorem to an asymmetric information economy with the equal treatment property. Sun and Yannelis (2007b) established this theorem in an economy with a continuum of agents and negligible asymmetric information. Below, we extend this result to the atomless ambiguous asymmetric information economy with a countable number of states of nature.

Theorem 4. Suppose Assumptions (E.3), (U.1), (U.3) and (I) hold. If the feasible step allocation $f$ is not in the $M C$ of the associated continuum economy $\mathcal{E}_{c}$, then for any $\alpha, 0<\alpha<1$, there exists a coalition $S$ such that $\mu(S)=\alpha$, which maximin blocks $f$.

Proof. See appendix.

\section{Equivalence Theorems}

Consider the finite agent economy $\mathcal{E}$, Hervés-Beloso et al. (2005b) provided two equivalence results for the Walrasian expectations equilibrium in terms of the private blocking power of the grand coalition, and Bhowmik and Cao (2012b) extended this result to an asymmetric information economy whose commodity space is a Banach lattice. We will follow this approach and characterize the maximin expectations equilibrium. The following two theorems correspond to Theorem 4.1 and 4.2 of Hervés-Beloso et al. (2005b), the proofs are omitted since the same technique can be followed here.

\footnotetext{
${ }^{13}$ Indeed we do not need Assumptions (E.1) and (U.2).
} 
For an allocation $x=\left\{x_{i}\right\}_{i \in I}$ and a vector $a=\left(a_{1}, \cdots, a_{s}\right) \in[0,1]^{s}$, consider the ambiguous asymmetric information economy $\mathcal{E}(a, x)$ which is identical with $\mathcal{E}$ except for the random initial endowment of each agent $i$ given by the convex combination $e_{i}\left(a_{i}, x_{i}\right)=a_{i} e_{i}+\left(1-a_{i}\right) x_{i}$.

Definition 6. An allocation $z$ is maximin dominated (or maximin blocked by the grand coalition) in the economy $\mathcal{E}(a, x)$ if there exists a feasible allocation $y$ in $\mathcal{E}(a, x)$ such that $V_{i}\left(y_{i}\right)>V_{i}\left(z_{i}\right)$ for every $i \in I$.

Theorem 5. The allocation $x$ is an $M E E$ in $\mathcal{E}$ if and only if $x$ is not a maximin dominated allocation in every economy $\mathcal{E}(a, x)$.

Definition 7. A coalition $S \subseteq I$ maximin blocks an allocation $x$ in the sense of Aubin via $y=\left\{y_{i}\right\}_{i \in S}$ if for all $i \in S$, there is $\alpha_{i} \in(0,1]$ such that $V_{i}\left(y_{i}\right)>V_{i}\left(x_{i}\right)$ and $\sum_{i \in S} \alpha_{i} y_{i} \leq \sum_{i \in S} \alpha_{i} e_{i}$. The Aubin maximin core is the set of all feasible allocations that cannot be maximin blocked by any coalition in the sense of Aubin. An allocation $x$ is called Aubin non-dominated if $x$ is not maximin blocked by the grand coalition in the sense of Aubin.

Theorem 6. The allocation $x$ is an $M E E$ in $\mathcal{E}$ if and only if $x$ is not a maximin dominated allocation in the sense of Aubin in the economy $\mathcal{E}$.

\section{Efficiency and Incentive Compatibility un- der Ambiguity}

\subsection{Efficiency and Incentive Compatibility}

First We recall the notion of coalitional incentive compatibility of Krasa and Yannelis (1994).

Definition 8. An allocation $x$ is said to be coalitional incentive compatible $(C B I C)$ if the following does not hold: there exist a coalition $C \subseteq I$ and two distinct states $a$ and $b$ such that

1. $\Pi_{i}(a)=\Pi_{i}(b)$ for all $i \notin C$;

2. $e_{i}(a)+x_{i}(b)-e_{i}(b) \in \mathbb{R}_{+}^{l}$ for all $i \in C$;

3. $u_{i}\left(a, y_{i}\right)>u_{i}\left(a, x_{i}\right)$ for all $i \in C$;

where for all $i \in C$,

$$
y_{i}(\omega)= \begin{cases}e_{i}(a)+x_{i}(b)-e_{i}(b) & \text { if } \omega=a \\ x_{i}(\omega) & \text { otherwise }\end{cases}
$$


Example 2. [Example 1 with Bayesian preference]

Suppose that agents are Bayesian expected utility maximizers, therefore all allocations are required to be private information measurable. The allocation $x_{1}=$ $(5,5,0)$ and $x_{2}=(5,0,5)$ is in the (non-free disposal) private core and it is incentive compatible. Indeed, it has been shown in Koutsougeras and Yannelis (1993) that private core allocations in non-free disposal economies are always CBIC provided that the utility functions are monotone and continuous.

This conclusion is not true in free disposal economies. Glycopantis et al. (2003) pointed out that private core and Walrasian expectations equilibrium allocations need not be incentive compatible in an economy with free disposal. In this example, $x_{1}=(4,4,1)$ and $x_{2}=(4,1,4)$ is a (free disposal) WEE allocation with the equilibrium price $p(a)=0$ and $p(b)=p(c)=\frac{1}{2}$, and hence in the (free disposal) private core. However, this allocation is not incentive compatible. Indeed, if agent 1 observes $\{a, b\}$, he has an incentive to report $c$ to become better off. Note that agent 2 cannot distinguish the state a from the state $c$. In particular, if the state a occurs and agent 1 reports state $c$, then his utility is

$$
u_{1}\left(e_{1}(a)+x_{1}(c)-e_{1}(c)\right)=u_{1}(5+1-0)=\sqrt{6}>\sqrt{4}=u_{1}\left(x_{1}(a)\right) .
$$

Hence, the free disposal WEE allocation is not incentive compatible.

\subsection{Maximin Incentive Compatibility}

In this subsection, we will prove that any maximin efficient allocation is maximin coalitional incentive compatible. The notion of maximin coalitional incentive compatibility have been introduced in de Castro et al. (2011) and de Castro et al. (2012), which is an extension of the coalitional incentive compatibility in Krasa and Yannelis (1994) and allows agents to have maximin preferences.

Definition 9. An allocation $x$ is said to be maximin coalitional incentive compatible $(M C I C)$ if the following does not hold: there exist a coalition $C \subseteq I$ and two distinct states $a$ and $b$ such that

1. $\Pi_{i}(a)=\Pi_{i}(b)$ for all $i \notin C$;

2. $e_{i}(a)+x_{i}(b)-e_{i}(b) \in \mathbb{R}_{+}^{l}$ for all $i \in C$;

3. $v_{i}\left(a, y_{i}\right)>v_{i}\left(a, x_{i}\right)$ for all $i \in C$;

where for all $i \in C$,

$$
y_{i}(\omega)= \begin{cases}e_{i}(a)+x_{i}(b)-e_{i}(b) & \text { if } \omega=a \\ x_{i}(\omega) & \text { otherwise }\end{cases}
$$


If $C$ is restricted to be a singleton, then it is individual maximin incentive compatible (MIC).

An allocation is said to be maximin coalitional incentive compatible if no coalition can misreport the realized states and make its members better off. Below, we revisit Example 2 and show that if agents are maximin expected utility maximizers, then the maximin core allocation in the above example is maximin incentive compatible.

Example 3. [Example 1 with maximin preference]

Now we assume that agents 1 and 2 are both maximin expected utility maximizers. As shown in Example 1, $x_{1}=(5,4,1)$ and $x_{2}=(5,1,4)$ is an MEE allocation, and hence in the maximin core. Now we check that this allocation is maximin incentive compatible.

Suppose state a occurs, if agent 1 truthfully reports, then his payoff is

$$
v_{1}\left(a, x_{1}\right)=\min \left\{u_{1}\left(a, x_{1}(a)\right), u_{1}\left(b, x_{1}(b)\right)\right\}=\min \{\sqrt{5}, \sqrt{4}\}=2 ;
$$

if agent 1 reports state $c$, then his payoff is

$$
\min \left\{u_{1}\left(a, e_{1}(a)+x_{1}(c)-e_{1}(c)\right), u_{1}\left(b, x_{1}(b)\right)\right\}=\min \{\sqrt{6}, \sqrt{4}\}=2 .
$$

Therefore, agent 1 has no incentive to misreport as he doesn't gain anything by misreporting. Similarly we could show that agent 2 also has no incentive to misreport.

In the following theorem we show that any maximin efficient allocation is maximin coalitional incentive compatible. If the state space is finite, this result has been established. For example, (1) de Castro et al. (2011) showed that any maximin interim efficient allocation is maximin coalitional incentive compatible; (2) de Castro and Yannelis (2010) showed that any maximin ex ante efficient allocation (which is called maximin efficient allocation therein) is maximin interim efficient. Below we extend their results to an economy with a countable number of states. For simplicity, we only prove the relation between maximin efficient allocation and maximin coalitional incentive compatibility, interested readers may check that (1) and (2) above are still valid.

Theorem 7. If Assumptions (E), (U) and (I) hold, then any maximin efficient allocation in $\mathcal{E}$ is $M C I C$.

Proof. See appendix.

Corollary 2. Under the conditions of Theorem 7, any MC or MEE allocation is maximin coalitional incentive compatible. 
Now we extend the notion of maximin coalitional incentive compatibility to the atomless economy $\mathcal{E}_{0}$ defined in Subsection 4.1.

Definition 10. An allocation $x$ in $\mathcal{E}_{0}$ is said to be maximin coalitional incentive compatible (MCIC) if the following does not hold: there exist a non-negligible subset $S \in \mathcal{T}$ and two distinct states $a$ and $b$ such that

1. $\Pi_{t}(a)=\Pi_{t}(b)$ for all $t \notin S$;

2. $e_{t}(a)+x_{t}(b)-e_{t}(b) \in \mathbb{R}_{+}^{l}$ for all $t \in S$;

3. $v_{t}\left(a, y_{t}\right)>v_{t}\left(a, x_{t}\right)$ for all $t \in S$;

where for all $t \in S$,

$$
y_{t}(\omega)= \begin{cases}e_{t}(a)+x_{t}(b)-e_{t}(b) & \text { if } \omega=a \\ x_{t}(\omega) & \text { otherwise }\end{cases}
$$

The following theorem is an extension of Theorem 7 to the economy $\mathcal{E}_{c}$ with the equal treatment property. We omit the proof since it is analogous to Theorem 7 .

Theorem 8. If Assumptions (E), (U) and (I) hold, and the step allocation $f$ in $\mathcal{E}_{c}$ is maximin efficient, then it is $M C I C$.

\section{Concluding Remarks}

We presented a new asymmetric information economy framework, where agents face ambiguity (i.e., they are MEU maximizers) and also the state space is not necessarily finite. This new set up allowed us to derive new core -Walras existence and equivalence results. It should be noted that contrary to the Bayesian asymmetric information economy framework, our core and Walrasian equilibrium concepts formulated in an ambiguous asymmetric information economy framework are now incentive compatible and obviously efficient. For this reason, we believe that our new results will be useful to other fields in economics.

In addition to the new conceptual results obtained in this paper, we also introduced a new way of proving theorems for an infinite (countable) number of states. Recall that even with one good the countable number of states brings us to an infinite dimensional set up. One way to prove infinite dimensional results in economics is to trace them in finite dimensions by truncating the dimensionality of the commodity space and then take limits to cover the full space (e.g. Bewley (1972)). In this paper we truncated the set of states and then allowed them to go 
to infinity. This way we avoided Banach space methods and made the arguments rather transparent and easier to read.

We would like to conclude by saying that the continuum of states and modeling perfect competition as in Sun and Yannelis (2007a,b) and Sun and Yannelis (2008) in the presence of ambiguity remains an open question and further research in this direction seems to be needed.

\section{Appendix}

\subsection{Truncated Economy}

Suppose that Assumption (I) holds.

$\mathcal{E}=\left\{(\Omega, \mathcal{F}) ;\left(\mathcal{F}_{i}, u_{i}, e_{i}, \pi_{i}\right): i \in I=\{1, \ldots, s\}\right\}$ is an ambiguous asymmetric information economy with a countable number of states. For every $n \in \mathbb{N}$, we define a truncated economy with a finite number of states as follow:

$$
\mathcal{E}^{n}=\left\{\left(\Omega^{n}, \mathcal{F}^{n}\right) ;\left(\mathcal{F}_{i}^{n}, u_{i}, e_{i}, \pi_{i}^{n}\right): i \in I=\{1, \ldots, s\}\right\},
$$

where $\Omega^{n}=F_{n}, \mathcal{F}^{n}$ is the restriction of $\mathcal{F}$ on $F_{n}$, i.e., $\mathcal{F}^{n}=\left\{E \subseteq F_{n}: E \in \mathcal{F}\right\}$. Similarly, $\mathcal{F}_{i}^{n}$ and $\Pi_{i}^{n}$ are restrictions of $\mathcal{F}_{i}$ and $\Pi_{i}$ on $F_{n}$ respectively. $\pi_{i}^{n}\left(E_{i}\right)=$ $\frac{\pi_{i}\left(E_{i}\right)}{\pi_{i}\left(F_{n}\right)}$ for every $i \in I$ and $E_{i} \in \mathcal{F}_{i}^{n} . u_{i}$ and $e_{i}$ are unchanged except that their domains are restricted on $\Omega^{n}$. For any allocation $x_{i}$ of agent $i$ in the economy $\mathcal{E}^{n}$, the maximin ex ante utility function is

$$
V_{i}^{n}\left(x_{i}\right)=\sum_{E_{i} \in \Pi_{i}^{n}}\left[\min _{\omega \in E_{i}} u_{i}\left(\omega, x_{i}(\omega)\right)\right] \pi_{i}^{n}\left(E_{i}\right) .
$$

Then $\mathcal{E}^{n}$ is a well defined ambiguous asymmetric information economy with finitely many states.

If $f$ is an allocation in the economy $\mathcal{E}$, then $f^{n}$ will be the allocation in the economy $\mathcal{E}^{n}$ such that $f^{n}$ is the restriction of $f$ on $\Omega^{n}$. Given any allocation $f^{n}$ in the economy $\mathcal{E}^{n}, f^{n, e}$ and $f^{n, 0}$ are two allocations in the economy $\mathcal{E}$ defined as follow: for every $i \in I$,

$$
f_{i}^{n, e}(\omega)=\left\{\begin{array}{ll}
f_{i}(\omega) & \text { if } \omega \in F_{n} \\
e_{i}(\omega) & \text { if } \omega \notin F_{n}
\end{array}, f_{i}^{n, 0}(\omega)=\left\{\begin{array}{ll}
f_{i}(\omega) & \text { if } \omega \in F_{n} \\
0 & \text { if } \omega \notin F_{n}
\end{array} .\right.\right.
$$

Similarly, given the price vector $p$ in the economy $\mathcal{E}, p^{n}$ is the restriction of $p$ on $F_{n}$ and $p^{n, 0}(\omega)=\left\{\begin{array}{ll}p^{n}(\omega) & \text { if } \omega \in F_{n} \\ 0 & \text { if } \omega \notin F_{n}\end{array}\right.$. 


\subsection{Proof of Section 2}

Proof of Proposition 1.

For any $i \in I$, we check that $V_{i}$ is uniformly continuous on $G$. Suppose $d\left(z^{k}, z\right) \rightarrow 0$ as $k \rightarrow \infty, z \in G$ and $z^{k} \in G$ for all $k$, we need to show $\left|V_{i}\left(z^{k}\right)-V_{i}(z)\right| \rightarrow$ 0, i.e., $\forall \epsilon>0, \exists k_{1}$ sufficiently large, $\forall k \geq k_{1},\left|V_{i}\left(z^{k}\right)-V_{i}(z)\right|<\epsilon$.

By Assumption (U.3), $\exists K>0$ such that $u_{i}(\omega, y(\omega)) \leq K$ for all $y \in G$ and $\omega \in \Omega$. Since $\cup_{m \in N} F_{m}=\Omega, \exists m_{0}$ sufficiently large, such that $\pi_{i}\left(F_{m_{0}}\right)>1-\frac{\epsilon}{2 K}$. $\Pi_{i}^{m_{0}}$ is the restriction of $\Pi_{i}$ on $F_{m_{0}}$.

Therefore:

$$
\begin{aligned}
\left|V_{i}\left(z^{k}\right)-V_{i}(z)\right| & =\left|\sum_{E_{i} \in \Pi_{i}}\left[\min _{\omega \in E_{i}} u_{i}\left(\omega, z^{k}(\omega)\right)\right] \pi_{i}\left(E_{i}\right)-\sum_{E_{i} \in \Pi_{i}}\left[\min _{\omega \in E_{i}} u_{i}(\omega, z(\omega))\right] \pi_{i}\left(E_{i}\right)\right| \\
& \leq\left|\sum_{E_{i} \notin \Pi_{i}^{m_{0}}}\left[\min _{\omega \in E_{i}} u_{i}\left(\omega, z^{k}(\omega)\right)-\min _{\omega \in E_{i}} u_{i}(\omega, z(\omega))\right] \pi_{i}\left(E_{i}\right)\right| \\
& +\left|\sum_{E_{i} \in \Pi_{i}^{m_{0}}}\left[\min _{\omega \in E_{i}} u_{i}\left(\omega, z^{k}(\omega)\right)-\min _{\omega \in E_{i}} u_{i}(\omega, z(\omega))\right] \pi_{i}\left(E_{i}\right)\right| .
\end{aligned}
$$

For the first term,

$$
\begin{aligned}
& \left|\sum_{E_{i} \notin \Pi_{i}^{m_{0}}}\left[\min _{\omega \in E_{i}} u_{i}\left(\omega, z^{k}(\omega)\right)-\min _{\omega \in E_{i}} u_{i}(\omega, z(\omega))\right] \pi_{i}\left(E_{i}\right)\right| \\
\leq & \sum_{E_{i} \notin \Pi_{i}^{m_{0}}} K \pi_{i}\left(E_{i}\right) \\
= & K \pi_{i}\left(F_{m_{0}}^{c}\right) \\
< & \frac{\epsilon}{2} .
\end{aligned}
$$

For any $\omega, u_{i}(\omega, \cdot)$ is continuous on the set $G_{0}=\left\{x \in \mathbb{R}_{+}^{l}: 0 \leq x(j) \leq\right.$ $M, j=1,2, \cdots, l$.$\} ; since G_{0}$ is compact, $u_{i}(\omega, \cdot)$ is uniformly continuous. Since $F_{m_{0}}$ has only finitely many states, $\exists \delta>0, \forall a, b \in G_{0}$, if $\|a-b\|<\delta$, then $\left|u_{i}(\omega, a)-u_{i}(\omega, b)\right|<\frac{\epsilon}{2}$ for all $\omega \in F_{m_{0}}$.

We claim that $\exists k_{1} \in \mathbb{N}$, such that $\forall k \geq k_{1},\left\|z^{k}(\omega)-z(\omega)\right\|<\delta$ for all $\omega \in F_{m_{0}}$.

Suppose the claim is not true, then $\forall k_{1} \in \mathbb{N}, \exists k \geq k_{1},\left\|z^{k}(\omega)-z(\omega)\right\| \geq \delta$ for some $\omega \in F_{m_{0}}$. Since $F_{m_{0}}$ has only finitely many states, $\exists \omega_{q_{0}} \in F_{m_{0}}$ and a sequence $\left\{k_{m}\right\}_{m \in \mathbb{N}}$, such that $\left\|z^{k_{m}}\left(\omega_{q_{0}}\right)-z\left(\omega_{q_{0}}\right)\right\| \geq \delta$ for all $k_{m}$. Thus $d\left(z^{k_{m}}, z\right)=$ $\sup _{t}\left[\frac{1}{t} \min \left\{\left\|z^{k_{m}}\left(\omega_{t}\right)-z\left(\omega_{t}\right)\right\|, 1\right\}\right] \geq \frac{1}{q_{0}} \min \left\{\left\|z^{k_{m}}\left(\omega_{q_{0}}\right)-z\left(\omega_{q_{0}}\right)\right\|, 1\right\} \geq \frac{1}{q_{0}} \min \{\delta, 1\}$, which contradicts the assumption that $d\left(z^{k}, z\right) \rightarrow 0$ as $k \rightarrow \infty$. Hence we prove the claim. 
For $k \geq k_{1},\left\|z^{k}(\omega)-z(\omega)\right\|<\delta$ for all $\omega \in F_{m_{0}}$, so $\left|u_{i}\left(\omega, z^{k}(\omega)\right)-u_{i}(\omega, z(\omega))\right|<$ $\frac{\epsilon}{2}$ for all $\omega \in F_{m_{0}}$. Therefore, for any $E_{i} \in \Pi_{i}^{m_{0}},\left|\min _{\omega \in E_{i}} u_{i}\left(\omega, z^{k}(\omega)\right)-\min _{\omega \in E_{i}} u_{i}(\omega, z(\omega))\right|<$ $\frac{\epsilon}{2}$, hence

$$
\begin{aligned}
& \left|\sum_{E_{i} \in \Pi_{i}^{m_{0}}}\left[\min _{\omega \in E_{i}} u_{i}\left(\omega, z^{k}(\omega)\right)-\min _{\omega \in E_{i}} u_{i}(\omega, z(\omega))\right] \pi_{i}\left(E_{i}\right)\right| \\
\leq & \sum_{E_{i} \in \Pi_{i}^{m_{0}}}\left|\min _{\omega \in E_{i}} u_{i}\left(\omega, z^{k}(\omega)\right)-\min _{\omega \in E_{i}} u_{i}(\omega, z(\omega))\right| \pi_{i}\left(E_{i}\right) \\
< & \frac{\epsilon}{2} \pi_{i}\left(F_{m_{0}}\right) \\
< & \frac{\epsilon}{2} .
\end{aligned}
$$

Summing up the two terms, $\left|V_{i}\left(z^{k}\right)-V_{i}(z)\right|<\epsilon$ for all $k \geq k_{1}$, we prove that $V_{i}(\cdot)$ is uniformly continuous.

\subsection{Proof of Section 3}

Proof of Lemma 2.

We will divide the proof into three parts. Firstly, we restrict the commodity space to be a compact set and show that the set of MEE is nonempty. Secondly, we relax the compactness assumption in step 1 and construct a sequence of economies, for each economy in this sequence we could get an equilibrium based on the results of step 1, then the sequence of equilibria has a convergent point. Thirdly, we prove that this convergent point is exactly the MEE for the original economy.

Let now $\Omega$ be a finite set, this restriction will be relaxed in Theorem 1 .

Step 1: This step is standard and we include it for the sake of completeness.

Let $X_{i}$ be the compact commodity space for agent $i$ such that $e_{i}(\omega)$ is an interior point of $X_{i}$ for any $i \in I$ and $\omega \in \Omega$.

For any price vector $q \in \triangle$, the budget set is $B_{i}(q)=\left\{y_{i} \in L_{i}: q \cdot y_{i} \leq\right.$ $q \cdot e_{i}, y_{i}(\omega) \in X$ for all $\left.\omega \in \Omega\right\}$. Since $e_{i}$ is an interior point of $X$, it is lowerhemicontinuous; moreover, $B_{i}(\cdot)$ has compact range and obviously has a closed graph (hence it is upper-hemicontinuous). Thus, $B_{i}(\cdot)$ is continuous.

Define the demand correspondence as follow: $D_{i}(q)=\left\{x_{i} \in B_{i}(q): V_{i}\left(x_{i}\right) \geq\right.$ $\left.V_{i}\left(y_{i}\right), \forall y_{i} \in B_{i}(q)\right\}$, where $q \in \triangle$. By Berge's maximum theorem, $D_{i}(\cdot)$ is upperhemicontinuous, nonempty and compact-valued. Moreover, $V_{i}$ is a concave function since $u_{i}$ is concave and $B_{i}(q)$ is convex, and therefore $D_{i}(q)$ is convex.

Define $Z(q)=\sum_{i \in I} D_{i}(q)-\sum_{i \in I} e_{i}$. Since $D_{i}(\cdot)$ is upper-hemicontinuous, nonempty, convex and compact-valued for any $i \in I$, so is $Z(\cdot)$. 
$\forall q \in \triangle, \forall z \in Z(q), \exists x_{i} \in D_{i}(q)$ for all $i \in I$, such that $z=\sum_{i \in I} x_{i}-\sum_{i \in I} e_{i}$. Since $x_{i} \in D_{i}(q) \subseteq B_{i}(q)$, so $q \cdot x_{i} \leq q \cdot e_{i}$ for all $i \in I$, thus $q \cdot z \leq 0$.

It follows from the Gale-Nikaido-Debreu lemma, $\exists p^{*} \in \triangle$, such that $Z\left(p^{*}\right) \cap$ $R_{-}^{m l} \neq \emptyset$, where $m$ is the number of states in $\Omega$. Suppose $z \in Z\left(p^{*}\right) \cap R_{-}^{m l}$, then $\exists x_{i} \in D_{i}(q)$ for all $i \in I$, such that $z=\sum_{i \in I} x_{i}-\sum_{i \in I} e_{i}$. This means that

- $\forall i \in I, x_{i}$ maximizes the ex ante utility $V_{i}$ subject to the budget set $B_{i}\left(p^{*}\right)$;

- $\sum_{i \in I} x_{i} \leq \sum_{i \in I} e_{i}$.

Step: 2: We now relax the compactness assumption.

For all $k \in \mathbb{N}$ and $i \in I$, let $X_{i}^{k}$ be the set:

$$
X_{i}^{k}=\left\{x: \sum_{h=1}^{l} x(\omega, h) \leq k \sum_{\omega^{\prime} \in \Omega} \sum_{h=1}^{l} e_{i}\left(\omega^{\prime}, h\right) \text { for all } \omega \in \Omega\right\} .
$$

Then $X_{i}^{k}$ is compact, convex and nonempty. Define $X^{k}=\prod_{i \in I} X_{i}^{k}$, by the proof in step 1 , for every $k$, there exists a sequence $\left(p^{k}, x^{k}\right) \in \Delta \times X^{k}$, such that for all $k \in \mathbb{N}$,

(i-k) for all $i \in I, x_{i}^{k} \in B_{i}\left(p^{k}\right)$;

(ii-k) for all $i \in I, V_{i}\left(x_{i}^{k}\right)=\max _{y_{i} \in B_{i}\left(p^{k}\right)} V_{i}\left(y_{i}\right)$;

(iii-k) $\sum_{i \in I} x_{i}^{k}(\omega) \leq \sum_{i \in I} e_{i}(\omega)$ for all $\omega \in \Omega$.

Define $Y=\left(\prod_{\omega \in \Omega} \prod_{h=1}^{l}\left[0, \sum_{i \in I} e_{i}(\omega, h)\right]\right)^{I}$, then $Y$ is compact and $x^{k} \in Y$ for each $k$. It means that there is a subsequence, say $\left\{\left(p^{k}, x^{k}\right)\right\}$, which converges to some $\left(p^{*}, x^{*}\right) \in \triangle \times Y$.

Moreover, $\forall i \in I, x_{i}^{k} \in B_{i}\left(p^{k}\right)$ for every $k$ implies that $x_{i}^{*} \in B_{i}\left(p^{*}\right)$, and $\sum_{i \in I} x_{i}^{k} \leq \sum_{i \in I} e_{i}$ for every $k$ implies that $\sum_{i \in I} x_{i}^{*} \leq \sum_{i \in I} e_{i}$. Because of the strong monotonicity assumption of the utility function, we get the equality $\sum_{i \in I} x_{i}^{*}=\sum_{i \in I} e_{i}$

Step 3: We check that $\left(p^{*}, x^{*}\right)$ is an MEE.

Suppose that there exist $i \in I$ and $y_{i} \in L_{i}$ such that $V_{i}\left(y_{i}\right)>V_{i}\left(x_{i}^{*}\right)$ and $y_{i} \in B_{i}\left(p^{*}\right)$, by the continuity of $u_{i}(\omega, \cdot)$ for every $\omega$, without loss of generality we may assume that $V_{i}\left(y_{i}\right)>V_{i}\left(x_{i}^{*}\right)$ and $y_{i} \cdot p^{*}<e_{i} \cdot p^{*}$.

Moreover, $x_{i}^{k} \rightarrow x_{i}^{*}$ implies that there exists a $k_{1}$, such that for $k>k_{1}, V_{i}\left(y_{i}\right)>$ $V_{i}\left(x_{i}^{k}\right)$. Because $V_{i}\left(x_{i}^{k}\right)=\max _{z_{i} \in B_{i}\left(p^{k}\right)} V_{i}\left(z_{i}\right)$, so $y_{i} \cdot p^{k}>e_{i} \cdot p^{k}$. Taking the limit, $y_{i} \cdot p^{*} \geq e_{i} \cdot p^{*}$, a contradiction.

Therefore, we have proved the existence of MEE, i.e., 
1. for all $i \in I, x_{i}^{*}$ maximizes $V_{i}(\cdot)$ in the budget set $B_{i}\left(p^{*}\right)$;

2. $\sum_{i \in I} x_{i}^{*}(\omega)=\sum_{i \in I} e_{i}(\omega)$ for all $\omega \in \Omega$.

\section{Proof of Theorem 1.}

For every $m \in \mathbb{N}, \mathcal{E}^{m}$ is a truncated economy of $\mathcal{E}$ defined in the subsection 8.1. By Lemma 2, there is an MEE for every economy $\mathcal{E}^{m}$, say $\left\{p^{m}, x^{m}\right\}$.

By Assumption (E.3), $\exists M>0$, such that $\forall \omega \in \Omega$ and $1 \leq j \leq l, \sum_{i \in I} e_{i}(\omega, j) \leq$ $M$. Define

$$
G=\left\{x \in\left(\mathbb{R}_{+}^{l}\right)^{\infty}: 0 \leq x(\omega, j) \leq M \text { for all } \omega \in \Omega \text { and } j \in=1,2, \cdots, l .\right\} .
$$

For any $m \in \mathbb{N}, i \in I, \omega \in \Omega$ and $j=1, \cdots, l, x_{i}^{m, e}(\omega, j) \leq \sum_{i \in I} e_{i}(\omega, j) \leq M$, thus $x_{i}^{m, e} \in G$.

Since $\triangle \times G^{I}$ is a compact set, $\left\{\left(p^{m, 0}, x^{m, e}\right)\right\}$ has a convergent subsequence, w.l.o.g, we assume that there exists an allocation $(x, p) \in \triangle \times G^{I}$ such that $\left(p^{m, 0}, x^{m, e}\right) \rightarrow(p, x)$ as $m \rightarrow \infty$. Moreover, it is easy to see that $x$ is feasible since $x^{m, e}$ is feasible for every $m$.

We verify that $(p, x)$ is an MEE.

First $x_{i} \in B_{i}(p)$, since $x_{i}^{m, e} \rightarrow x_{i}, p^{m, 0} \rightarrow p$ and $x_{i}^{m, e} \in B_{i}\left(p^{m, 0}\right)$.

For any $y \in B_{i}(p)$, let $y^{k}$ be the restriction of $y$ on $F_{k}$, then $d\left(y^{k, 0}, y\right) \rightarrow 0$ as $k \rightarrow \infty$, hence $V_{i}\left(y^{k, 0}\right) \rightarrow V_{i}(y)$.

For every $k, \exists m_{k}$ sufficiently large such that $m_{k} \geq k$ and $y^{k}$ is in $i$ 's budget set ${ }^{14} B_{i}\left(p^{m_{k}}\right)$ in the truncated economy $\mathcal{E}^{m_{k}}$, since $x_{i}^{m_{k}}$ is the maximal point,

$$
\sum_{E_{i} \in \Pi_{i}^{m_{k}}}\left[\min _{\omega \in E_{i}} u_{i}\left(\omega, y^{k}(\omega)\right)\right] \pi_{i}^{m_{k}}\left(E_{i}\right) \leq \sum_{E_{i} \in \Pi_{i}^{m_{k}}}\left[\min _{\omega \in E_{i}} u_{i}\left(\omega, x_{i}^{m_{k}}(\omega)\right)\right] \pi_{i}^{m_{k}}\left(E_{i}\right),
$$

so

$$
\sum_{E_{i} \in \Pi_{i}^{m_{k}}}\left[\min _{\omega \in E_{i}} u_{i}\left(\omega, y^{k}(\omega)\right)\right] \pi_{i}\left(E_{i}\right) \leq \sum_{E_{i} \in \Pi_{i}^{m_{k}}}\left[\min _{\omega \in E_{i}} u_{i}\left(\omega, x_{i}^{m_{k}}(\omega)\right)\right] \pi_{i}\left(E_{i}\right),
$$

which implies $V_{i}\left(y^{k, 0}\right) \leq V_{i}\left(x_{i}^{m_{k}, e}\right)$ for every $k$.

Then $V_{i}\left(x_{i}\right)=\lim _{k \rightarrow \infty} V_{i}\left(x_{i}^{m_{k}, e}\right) \geq \lim \sup _{k \rightarrow \infty} V_{i}\left(y^{k, 0}\right)=V_{i}(y), x_{i}$ is the agent $i$ 's maximal point in his budget set.

The proof completes.

\footnotetext{
${ }^{14}$ Here we slightly abuse the notation, $y^{k}$ is now considered as an allocation of the economy $\mathcal{E}^{m_{k}}$, such that $y^{k}(\omega)=0$ for $\omega \in F_{m_{k}} \backslash F_{k}$.
} 


\subsection{Proof of Section 4}

\subsubsection{Proof of Subsection 4.3}

Definition 11. A feasible allocation $x$ is said to be an $\epsilon$-maximin core allocation $(\epsilon-M C)$ in the economy $\mathcal{E}_{c}$, if there do not exist a coalition $C \subseteq T, \mu(C)>0$, and an allocation $y$ such that

(i) $V_{t}\left(y_{t}\right)>V_{t}\left(x_{t}\right)+\epsilon$ for $\mu$-almost all $t \in C$;

(ii) $\int_{C} y(t, \omega) \mathrm{d} \mu=\int_{C} e(t, \omega) \mathrm{d} \mu$ for all $\omega \in \Omega$.

For any allocation $x$, agent $t \in T$ and $\epsilon>0$, let $P_{t}^{\epsilon}\left(x_{t}\right)=\left\{y \in L_{t}: V_{t}(y)>\right.$ $\left.V_{t}\left(x_{t}\right)+\epsilon\right\}$ and $P_{t}\left(x_{t}\right)=\left\{y \in L_{t}: V_{t}(y)>V_{t}\left(x_{t}\right)\right\}$. Throughout this subsection, we assume that the step allocation $f$ is in the maximin core of the economy $\mathcal{E}_{c}$. $\phi(t)=P_{t}\left(f_{t}\right) \cup\left\{e_{t}\right\}$ and $\phi^{m}(t)=P_{t}^{\frac{1}{m}}\left(f_{t}^{m}\right) \cup\left\{e_{t}^{m}\right\}$, where $f_{t}^{m}$ and $e_{t}^{m}$ are the restrictions of $f_{t}$ and $e_{t}$ in the truncated economy $\mathcal{E}_{c}^{m}$.

Lemma 3. Suppose Assumptions (E), (U) and (I) hold. For any $\epsilon>0$, there exists $M$ such that for every $m>M, f^{m}$ is an $\epsilon$-MC allocation in the truncated economy $\mathcal{E}_{c}^{m}$.

Proof. By Assumptions (E) and (U), $\exists K>0$, such that $u_{t}\left(\omega, f_{t}(\omega)\right)<K$ for every $\omega \in \Omega$ and $t \in T$. By Assumption (I), $\exists M>0, \frac{\pi_{i}\left(F_{M}^{C}\right)}{\pi_{i}\left(F_{M}\right)}<\frac{\epsilon}{K}, \forall i \in I$.

For any $m>M$,

$$
\begin{aligned}
& V_{t}\left(f_{t}\right) \\
= & \sum_{E \in \Pi_{t}}\left[\min _{\omega \in E} u_{t}\left(\omega, f_{t}(\omega)\right)\right] \pi_{t}(E) \\
= & \sum_{E \in \Pi_{t}^{m}}\left[\min _{\omega \in E} u_{t}\left(\omega, f_{t}(\omega)\right)\right] \pi_{t}(E)+\sum_{E \notin \Pi_{t}^{m}}\left[\min _{\omega \in E} u_{t}\left(\omega, f_{t}(\omega)\right)\right] \pi_{t}(E) \\
< & \sum_{E \in \Pi_{t}^{m}}\left[\min _{\omega \in E} u_{t}\left(\omega, f_{t}(\omega)\right)\right] \pi_{t}(E)+K \pi_{t}\left(F_{m}^{C}\right) \\
< & \pi_{t}\left(F_{m}\right) V_{t}^{m}\left(f_{t}^{m}\right)+\epsilon \pi_{t}\left(F_{m}\right) .
\end{aligned}
$$

Then fix $m>M$, suppose $f^{m}$ is not an $\epsilon$-MC allocation in the truncated economy $\mathcal{E}_{c}^{m}$, then there exists a coalition $C \subseteq T, \mu(C)>0$, and an allocation $g$ such that

(i) $V_{t}^{m}\left(g_{t}\right)>V_{t}^{m}\left(f_{t}^{m}\right)+\epsilon$ for almost all $t \in C$;

(ii) $\int_{C} g(t, \omega) \mathrm{d} \mu=\int_{C} e(t, \omega) \mathrm{d} \mu$ for all $\omega \in \Omega^{m}$.

Define

$$
z(t, \omega)= \begin{cases}g(t, \omega), & \text { if } \omega \in \Omega^{m} \\ e(t, \omega), & \text { if } \omega \notin \Omega^{m}\end{cases}
$$


Then in the economy $\mathcal{E}_{c}, V_{t}(z(t))>\pi_{t}\left(F_{m}\right) V_{t}^{m}\left(g_{t}\right)>\pi_{t}\left(F_{m}\right)\left[V_{t}^{m}\left(f_{t}^{m}\right)+\epsilon\right]>V_{t}\left(f_{t}\right)$. If $\omega \in \Omega^{m}, \int_{C} z(t, \omega) \mathrm{d} \mu=\int_{C} g(t, \omega) \mathrm{d} \mu=\int_{C} e(t, \omega) \mathrm{d} \mu$; if $\omega \notin \Omega^{m}, \int_{C} z(t, \omega) \mathrm{d} \mu=$ $\int_{C} e(t, \omega) \mathrm{d} \mu$. The coalition $C$ maximin blocks $f$ via $z$, a contradiction.

Lemma 4. Suppose Assumptions (E), (U) and (I) hold. If $p^{m} \cdot a \geq p^{m} \cdot \int_{T} e_{t}^{m} \mathrm{~d} \mu$ for every $a \in \int_{T} \phi^{m}(t) \mathrm{d} \mu$ and $p^{m, 0} \rightarrow p$ as $m \rightarrow \infty$, then $p \cdot b \geq p \cdot \int_{T} e_{t} \mathrm{~d} \mu$ for every $b$ such that $b(\omega)=\int_{T} g(t, \omega) \mathrm{d} \mu$ for some $g(t) \in \phi(t)$.

Note that $\int_{T} e_{t}^{m} \mathrm{~d} \mu$ is well defined since it is only finite dimensional, but $\int_{T} e_{t} \mathrm{~d} \mu$ is not the Bochner integral: $p \cdot \int_{T} e_{t} \mathrm{~d} \mu$ denotes $\sum_{\omega \in \Omega} p(\omega) \cdot \int_{T} e(t, \omega) \mathrm{d} \mu$.

Proof. By way of contradiction, suppose that there exists an allocation $g$ such that $g(t) \in \phi(t)$ for every $t \in T, b(\omega)=\int_{T} g(t, \omega) \mathrm{d} \mu$ for any $\omega$ and $p \cdot b<p \cdot \int_{T} e_{t} \mathrm{~d} \mu$.

We claim that for every $t \in T, \exists M_{t}>0, \forall m \geq M_{t}, g^{m}(t) \in \phi^{m}(t)$ in the truncated economy $\mathcal{E}_{c}^{m}$.

Define $E_{0}=\{t \in T: g(t)=e(t)\}$. If $t \in E_{0}, g^{m}(t)=e^{m}(t) \in \phi^{m}(t)$ for any $m$.

Otherwise, let $\epsilon_{t}=V_{t}(g(t))-V_{t}(f(t))>0$. In the proof of Lemma 3 we have shown that $\exists K>0$ such that $u_{t}\left(\omega, f_{t}(\omega)\right)<K$ for every $\omega \in \Omega$ and $t \in T$. Then there exist $M_{t}^{1}$ and $M_{t}^{2}$ such that $\frac{\pi_{t}\left(F_{M_{t}^{1}}\right)}{\pi_{t}\left(F_{M_{t}^{1}}\right)}<\frac{\epsilon_{t}}{2 K}$ and $\frac{1}{\pi_{t}\left(F_{\left.M_{t}^{2}\right)}\right.}-\frac{1}{2}>\frac{1}{M_{t}^{2} \cdot \epsilon_{t}}$. Let $M_{t}=\max \left\{M_{t}^{1}, M_{t}^{2}\right\}$. For $m \geq M_{t}$,

$$
\begin{aligned}
& V_{t}^{m}\left(g^{m}(t)\right)-V_{t}^{m}\left(f^{m}(t)\right) \\
= & \sum_{E \in \Pi_{t}^{m}}\left[\min _{\omega \in E} u_{t}\left(\omega, g_{t}^{m}(\omega)\right)\right] \pi_{t}^{m}(E)-\sum_{E \in \Pi_{t}^{m}}\left[\min _{\omega \in E} u_{t}\left(\omega, f_{t}^{m}(\omega)\right)\right] \pi_{t}^{m}(E) \\
= & \frac{1}{\pi_{t}\left(F_{m}\right)}\left[\sum_{E \in \Pi_{t}}\left[\min _{\omega \in E} u_{t}\left(\omega, g_{t}(\omega)\right)\right] \pi_{t}(E)-\sum_{E \notin \Pi_{t}^{m}}\left[\min _{\omega \in E} u_{t}\left(\omega, g_{t}(\omega)\right)\right] \pi_{t}(E)\right. \\
- & \left.\sum_{E \in \Pi_{t}}\left[\min _{\omega \in E} u_{t}\left(\omega, f_{t}(\omega)\right)\right] \pi_{t}(E)+\sum_{E \notin \Pi_{t}^{m}}\left[\min _{\omega \in E} u_{t}\left(\omega, f_{t}(\omega)\right)\right] \pi_{t}(E)\right] \\
> & \frac{1}{\pi_{t}\left(F_{m}\right)}\left[\sum_{E \in \Pi_{t}}\left[\min _{\omega \in E} u_{t}\left(\omega, g_{t}(\omega)\right)\right] \pi_{t}^{m}(E)-\sum_{E \in \Pi_{t}}\left[\min _{\omega \in E} u_{t}\left(\omega, f_{t}(\omega)\right)\right] \pi_{t}^{m}(E)-K \pi_{t}\left(F_{m}^{C}\right)\right] \\
> & \frac{1}{\pi_{t}\left(F_{m}\right)}\left[V_{t}(g(t))-V_{t}(f(t))\right]-\frac{\epsilon_{t}}{2} \\
> & \frac{1}{m} .
\end{aligned}
$$

Therefore, $g^{m}(t) \in \phi^{m}(t)$.

Define $E_{m}=\left\{t \in T: g^{k}(t) \in \phi^{k}(t)\right.$ for all $\left.k \geq m\right\}$, then $E_{m} \subseteq E_{m+1}$ and $\cup_{m=0}^{\infty} E_{m}=T$. For $m \geq 1$, define

$$
h^{m}(t)= \begin{cases}g^{m}(t), & t \in E_{m} \\ e^{m}(t), & t \notin E_{m}\end{cases}
$$


then $h^{m}(t) \in \phi^{m}(t)$ for all $t \in T$.

Because $h^{m, e}(t) \rightarrow g(t) \mu$-a.s. and $p^{m, 0} \rightarrow p$, so $p^{m} \cdot\left(h^{m}(t)-e^{m}(t)\right)=p^{m, 0}$. $\left(h^{m, e}(t)-e(t)\right) \rightarrow p \cdot\left(g_{t}-e_{t}\right) \mu$-a.s. We have

$$
\begin{aligned}
p^{m} \cdot \int_{T}\left(h^{m}(t)-e^{m}(t)\right) \mathrm{d} \mu & =\int_{T} p^{m} \cdot\left(h^{m}(t)-e^{m}(t)\right) \mathrm{d} \mu \rightarrow \int_{T} p \cdot(g(t)-e(t)) \mathrm{d} \mu \\
& =p \cdot \int_{T}(g(t)-e(t)) \mathrm{d} \mu=p \cdot\left(b-\int_{T} e(t) \mathrm{d} \mu\right),
\end{aligned}
$$

The first equality holds since $p^{m}, h^{m}$ and $e^{m}$ can be regarded as finite dimensional mappings, the convergence is due to the Lebesgue dominated convergence theorem, the second equality is due to the Fubini theorem. Thus there exists some $M>0$ such that for any $m>M, p^{m} \cdot\left(\int_{T} h^{m}(t) \mathrm{d} \mu-\int_{T} e^{m}(t) \mathrm{d} \mu\right)<0$, a contradiction.

Proof of Theorem 3.

One direction is obvious, we only need to prove that the $\mathrm{MC}$ allocation is also an MEE allocation.

Suppose that $f$ is an MC allocation, by Lemma 3, for sufficiently large $m \in \mathbb{N}$, $f^{m}$ is a $\frac{1}{m}$-MC allocation in the truncated economy $\mathcal{E}_{c}^{m}$, then the set $\int_{T} \phi^{m}(t) \mathrm{d} \mu$ is nonempty and convex by Lyapunov's theorem.

Define $L_{++}$to be the function from $\Omega^{m}$ to $R_{++}^{l}$, we claim that $\left[\int_{T} e_{t}^{m} \mathrm{~d} \mu-L_{++}\right] \cap$ $\int_{T} \phi^{m} \mathrm{~d} \mu=\emptyset$. Otherwise, there exists $z \in L_{++}$and an integrable function $g^{m}(t) \in \phi^{m}(t)$ for $\mu$-a.e. $t \in T$, such that

$$
\int_{T} e_{t}^{m} \mathrm{~d} \mu-z=\int_{T} g^{m}(t) \mathrm{d} \mu
$$

Define $S=\left\{t \in T: g^{m}(t) \in P_{t}^{\frac{1}{m}}\left(f_{t}^{m}\right)\right\}$, then $g^{m}(t)=e_{t}^{m}$ for $\mu$-a.e. $t \in T \backslash S$ and

$$
\int_{S} e_{t}^{m} \mathrm{~d} \mu-z=\int_{S} g^{m}(t) \mathrm{d} \mu
$$

Since $z \neq 0$, we have $\mu(S)>0$. Define $h^{m}(t)=g^{m}(t)+\frac{1}{\mu(S)} z$, then $\int_{S} e_{t}^{m} \mathrm{~d} \mu=$ $\int_{S} h^{m}(t) \mathrm{d} \mu$ and $h^{m}(t) \in \phi^{m}(t)$ for $\mu$-a.e. $t \in S$ because of the monotonicity of the preference. This is a contradiction.

By the separation theorem there is a nonzero linear functional $\psi$, such that $\psi(a) \geq \psi\left(\int_{T} e_{t}^{m} \mathrm{~d} \mu\right)-\psi(z)$ for every $a \in \int_{T} \phi_{t}^{m} \mathrm{~d} \mu$ and $z \in L_{++}$. Since $z$ is arbitrary, $\psi(a) \geq \psi\left(\int_{T} e_{t}^{m} \mathrm{~d} \mu\right)$ for every $a \in \int_{T} \phi_{t}^{m} \mathrm{~d} \mu$; since $\int_{T} e_{t}^{m} \mathrm{~d} \mu \in \int_{T} \phi_{t}^{m} \mathrm{~d} \mu$, $\psi(z) \geq 0$ for every $z \in L_{++}$. There exists a $p^{m} \neq 0$ such that $\psi(a)=p^{m} \cdot a$. Since $\psi(z) \geq 0$ for every $z \in L_{++}, p^{m} \geq 0$. Then $p^{m} \cdot a \geq p^{m} \cdot \int_{T} e_{t}^{m} \mathrm{~d} \mu$ for every $a \in \int_{T} \phi_{t}^{m} \mathrm{~d} \mu$. Since $p^{m, 0} \in \triangle$ for every $m$ and $\triangle$ is compact, $\exists p \in \triangle$ which is a convergent point of $\left\{p^{m, 0}\right\}_{m \in \mathbb{N}}$. By Lemma $4, p \cdot b \geq p \cdot \int_{T} e_{t} \mathrm{~d} \mu$ for every 
$b \in \int_{T} \phi(t) \mathrm{d} \mu$.

Following the standard arguments in Hildenbrand (1974), we can show that $(f, p)$ is an MEE.

\subsubsection{Proof of Subsection 4.4}

Proof of Theorem 4.

Suppose that $f$ is maximin blocked by the coalition $A$ via $g^{\prime}$, then $\int_{A} g^{\prime}(t, \omega) \mathrm{d} \mu=$ $\int_{A} e(t, \omega) \mathrm{d} \mu$ for every $\omega \in \Omega$ and $V_{t}\left(g_{t}^{\prime}\right)>V_{t}\left(f_{t}\right)$ for every $t \in A$. Define $A_{i}=A \cap T_{i}$, for each $\omega \in \Omega$, let $g_{i}(\omega)=\frac{1}{\mu\left(A_{i}\right)} \int_{A_{i}} g_{t}^{\prime}(\omega) \mathrm{d} \mu$; suppose $g$ is defined as $g(t, \cdot)=g_{i}$ when $t \in A_{i}$. Then $\int_{A} g(t, \omega) \mathrm{d} \mu=\int_{A} e(t, \omega) \mathrm{d} \mu$ and $V_{i}\left(g_{t}\right)>V_{i}\left(f_{t}\right)$ for every $t \in A_{i}$ by the concavity of $V_{i}(\cdot)$.

Because of the uniform continuity of $V_{i}(\cdot), g_{t}^{m, 0}$ converges to $g_{t}$ as $m \rightarrow \infty$; thus there exists some $M>0$ such that for every $m \geq M, V_{t}\left(g_{t}^{m, 0}\right)>V_{t}\left(f_{t}\right)$ for every $t \in A_{i}$ with $\mu\left(A_{i}\right)>0$, and $\int_{A} g_{t}^{m, 0}(\omega) \mathrm{d} \mu=\int_{A} e_{t}(\omega) \mathrm{d} \mu$ for every $\omega \in F_{m}$.

Fix $m \geq M$, consider the finite dimensional atomless vector measure restricted to $A$, for $S \subseteq A$,

$$
\eta(S)=\left(\int_{S}\left(g_{t}^{m}(\cdot)-e_{t}^{m}(\cdot)\right) \mathrm{d} \mu, \mu(S)\right) .
$$

Applying Lyapunov's theorem to $\eta$, we obtain that for any $\alpha \in(0,1)$, there exists a coalition $B \subseteq A$, with $\mu(B)=\alpha \mu(A)$ and $\int_{B}\left(g_{t}^{m}(\omega)-e_{t}^{m}(\omega)\right) \mathrm{d} \mu=\alpha \int_{A}\left(g_{t}^{m}(\omega)-\right.$ $\left.e_{t}^{m}(\omega)\right) \mathrm{d} \mu=0$ for every $\omega \in F_{m}$. Let

$$
h_{t}(\omega)= \begin{cases}g_{t}^{m}(\omega), & \text { if } \omega \in F_{m} ; \\ \frac{1}{\mu(B)} \int_{B} e_{t}(\omega) \mathrm{d} \mu, & \text { if } \omega \notin F_{m}\end{cases}
$$

Then if $\omega \in F_{m}$, then $\int_{B} h_{t}(\omega) \mathrm{d} \mu=\int_{B} g_{t}^{m}(\omega) \mathrm{d} \mu=\int_{B} e_{t}(\omega) \mathrm{d} \mu$; if $\omega \notin F_{m}$, then $\int_{B} h_{t}(\omega) \mathrm{d} \mu=\int_{B}\left(\frac{1}{\mu(B)} \int_{B} e_{t}(\omega) \mathrm{d} \mu\right) \mathrm{d} \mu=\int_{B} e_{t}(\omega) \mathrm{d} \mu . B_{i}=B \cap T_{i}$, for every $i$ with $\mu\left(B_{i}\right)>0, V_{t}\left(h_{t}\right) \geq V_{t}\left(g_{t}^{m, 0}\right)>V_{t}\left(f_{t}\right)$ for $t \in B_{i}$, the coalition $B$ maximin blocks $f$ via $h$.

If $\mu(A)=1$, we are done; otherwise $\mu(T \backslash A)>0$. Since $V_{i}(\cdot)$ is continuous on the set of feasible allocations for every $i \in I$, we can choose $g$ such that $\frac{1}{\mu(A)} \int_{A}(e(t, \omega)-g(t, \omega)) \mathrm{d} \mu=z(\omega) \gg 0$ for every $\omega \in \Omega$ and $V_{t}\left(g_{t}\right)>V_{t}\left(f_{t}\right)$ for every $t \in A_{i}$.

Given $\alpha>0$, consider the allocation $g_{\alpha}(t, \omega)=\alpha g(t, \omega)+(1-\alpha) f(t, \omega)$. By concavity of $V_{t}(\cdot), V_{t}\left(g_{\alpha}(t)\right)>V_{t}(f(t))$ for every $t \in A$. By the uniform continuity of $V_{i}(\cdot)$, there exists some $M_{1}>0$ such that for every $m \geq M_{1}, V_{t}\left(g_{\alpha}^{m, e}(t)\right)>V_{t}\left(f_{t}\right)$ for every $t \in A$.

Consider $\bar{g}_{t}(\omega)=f_{t}(\omega)+\frac{\alpha \mu(A)}{\mu(L \backslash A)} z(\omega)$, then $V_{t}\left(\bar{g}_{t}\right)>V_{t}\left(f_{t}\right)$ by monotonicity. By the uniform continuity, there exists some $M_{2}>0$ such that for $m \geq M_{2}$, 
$V_{t}\left(\bar{g}_{t}^{m, e}\right)>V_{t}\left(f_{t}\right)$ for every $t$.

Fix $M>\max \left\{M_{1}, M_{2}\right\}$, consider the finite dimensional vector measure $\nu$ restricted to $T \backslash A$, for $C \subseteq T \backslash A$,

$$
\nu(C)=\left(\mu(C), \int_{C}\left(e_{t}^{M}(\cdot)-f_{t}^{M}(\cdot)\right) \mathrm{d} \mu\right) .
$$

Applying Lyapunov's theorem to $\nu$, for $\alpha>0$, there exists a coalition $B \subseteq T \backslash A$, such that

1. $\mu(B)=(1-\alpha) \mu(T \backslash A)$;

2. $\int_{B}\left(e_{t}^{M}(\cdot)-f_{t}^{M}(\cdot)\right) \mathrm{d} \mu=(1-\alpha) \int_{T \backslash A}\left(e_{t}^{M}(\cdot)-f_{t}^{M}(\cdot)\right) \mathrm{d} \mu$.

Consider the coalition $S=A \cup B$, then $\mu(S)=\mu(A)+(1-\alpha) \mu(T \backslash A)$. Define the allocation $y$ from $S \times \Omega$ to $\mathbb{R}_{+}^{l}$ as follow:

$$
y_{t}(\omega)= \begin{cases}g_{\alpha}(t, \omega), & \text { if } \omega \in \Omega^{M} \text { and } t \in A \\ f_{t}(\omega)+\frac{\alpha \mu(A)}{\mu(B)} z(\omega), & \text { if } \omega \in \Omega^{M} \text { and } t \in B \\ \frac{1}{\mu(S)} \int_{S} e_{t}(\omega) \mathrm{d} \mu, & \text { if } \omega \notin \Omega^{M} .\end{cases}
$$

If $t \in A, V_{t}\left(y_{t}\right)=V_{t}\left(g_{\alpha}^{M, e}(t)\right)>V_{t}\left(f_{t}\right)$. If $t \in B$, since $f_{t}(\omega)+\frac{\alpha \mu(A)}{\mu(B)} z(\omega) \geq$ $f_{t}(\omega)+\frac{\alpha \mu(A)}{\mu(T \backslash A)} z(\omega)=\bar{g}_{t}(\omega), V_{t}\left(y_{t}\right) \geq V_{t}\left(\bar{g}_{t}^{M, e}\right)>V_{t}\left(f_{t}\right)$. We only need to check that $y$ is feasible.

If $\omega \in \Omega^{M}$

$$
\begin{aligned}
& \int_{S} y(t, \omega) \mathrm{d} \mu \\
= & \int_{A} g_{\alpha}(t, \omega) \mathrm{d} \mu+\int_{B}\left(f(t, \omega)+\frac{\alpha \mu(A)}{\mu(B)} z(\omega)\right) \mathrm{d} \mu
\end{aligned}
$$$$
=\int_{A}(\alpha g(t, \omega)+(1-\alpha) f(t, \omega)) \mathrm{d} \mu+\int_{B} f(t, \omega) \mathrm{d} \mu+\alpha \mu(A) z(\omega)
$$$$
=\alpha \int_{A} e(t, \omega) \mathrm{d} \mu+\int_{A}(1-\alpha) f(t, \omega) \mathrm{d} \mu+\int_{B} e(t, \omega) \mathrm{d} \mu-(1-\alpha) \int_{T \backslash A}(e(t, \omega)-f(t, \omega)) \mathrm{d} \mu
$$$$
=\alpha \int_{A} e(t, \omega) \mathrm{d} \mu+\int_{B} e(t, \omega) \mathrm{d} \mu+(1-\alpha) \int_{A} e(t, \omega) \mathrm{d} \mu
$$

$=\int_{S} e(t, \omega) \mathrm{d} \mu ;$

If $\omega \notin \Omega^{M}$,

$$
\begin{aligned}
& \int_{S} y(t, \omega) \mathrm{d} \mu \\
= & \int_{S}\left(\frac{1}{\mu(S)} \int_{S} e(t, \omega) \mathrm{d} \mu\right) \mathrm{d} \mu
\end{aligned}
$$




$$
=\int_{S} e(t, \omega) \mathrm{d} \mu
$$

Therefore, the coalition $S$ maximin blocks the allocation $f$ via $y$, and the proof is complete.

\subsection{Proof of Section 6}

Proof of Theorem $\%$.

Suppose $\left\{x_{i}\right\}_{i \in I}$ is an maximin efficient allocation and not MCIC, then there exist a coalition $C$ and two states $a$ and $b$ such that: $v_{i}\left(a, y_{i}\right)>v_{i}\left(a, x_{i}\right)$ for all $i \in C$, where

$$
y_{i}(\omega)= \begin{cases}e_{i}(a)+x_{i}(b)-e_{i}(b) & \text { if } \omega=a, \\ x_{i}(\omega) & \text { otherwise. }\end{cases}
$$

Extending the allocation $y$ for all agents by letting

$$
y_{i}(\omega)= \begin{cases}e_{i}(a)+x_{i}(b)-e_{i}(b) & \text { if } \omega=a \\ x_{i}(\omega) & \text { otherwise }\end{cases}
$$

for all $i \in I$.

First we check that $\left\{y_{i}\right\}_{i \in I}$ is feasible.

(i) If $\omega \neq a, y_{i}(\omega)=x_{i}(\omega)$ for all $i \in I$, so $\sum_{i \in I} y_{i}(\omega)=\sum_{i \in I} x_{i}(\omega)=$ $\sum_{i \in I} e_{i}(\omega)$;

(ii) If $\omega=a, \sum_{i \in I} y_{i}(a)=\sum_{i \in I} e_{i}(a)+\sum_{i \in I} x_{i}(b)-\sum_{i \in I} e_{i}(b)=\sum_{i \in I} e_{i}(a)$, since $\sum_{i \in I} e_{i}(b)=\sum_{i \in I} x_{i}(b)$.

Second, we show that all the agents in $C$ are better off and all other agents are at least the same if taking the allocation $y$ instead of $x$.

For $i \in C$ and $E_{i} \in \Pi_{i}$, if $a \notin E_{i}$, then for any $\omega \in E_{i}, v_{i}\left(\omega, y_{i}\right)=v_{i}\left(\omega, x_{i}\right)$, because $y_{i}(\omega)=x_{i}(\omega)$ for all $\omega \in E_{i}$; if $a \in E_{i}$, then for any $\omega \in E_{i}, v_{i}\left(\omega, y_{i}\right)>$ $v_{i}\left(\omega, x_{i}\right)$ because of our assumption; therefore $V_{i}\left(y_{i}\right)=\sum_{E_{i} \in \Pi_{i}} v_{i}\left(E_{i}, y_{i}\right) \pi_{i}\left(E_{i}\right)>$ $\sum_{E_{i} \in \Pi_{i}} v_{i}\left(E_{i}, x_{i}\right) \pi_{i}\left(E_{i}\right)=V_{i}\left(x_{i}\right)$ for all $i \in C$.

For $i \notin C$ and $E_{i} \in \Pi_{i}$, if $a \notin E_{i}, v_{i}\left(E_{i}, y_{i}\right)=v_{i}\left(E_{i}, x_{i}\right)$, because $y_{i}(\omega)=x_{i}(\omega)$ for all $\omega \in E_{i}$; if $a \in E_{i}$, then $b \in E_{i}$ and $e_{i}(a)=e_{i}(b)$, hence $u_{i}\left(a, y_{i}(a)\right)=$ $u_{i}\left(a, e_{i}(a)+x_{i}(b)-e_{i}(b)\right)=u_{i}\left(a, x_{i}(b)\right)=u_{i}\left(b, y_{i}(b)\right)$.

$$
\begin{aligned}
v_{i}\left(E_{i}, y_{i}\right) & =\min \left(u_{i}\left(a, y_{i}(a)\right), \min _{\omega \neq a, \omega \in E_{i}} u_{i}\left(\omega, y_{i}(\omega)\right)\right) \\
& =\min \left(u_{i}\left(b, y_{i}(b)\right), \min _{\omega \neq a, \omega \in E_{i}} u_{i}\left(\omega, y_{i}(\omega)\right)\right) \\
& =\min _{\omega \neq a, \omega \in E_{i}} u_{i}\left(\omega, x_{i}(\omega)\right)
\end{aligned}
$$




$$
\begin{aligned}
& \geq \min _{\omega \in E_{i}} u_{i}\left(\omega, x_{i}(\omega)\right) \\
& =v_{i}\left(E_{i}, x_{i}\right) .
\end{aligned}
$$

Then $V_{i}\left(y_{i}\right)=\sum_{E_{i} \in \Pi_{i}} v_{i}\left(E_{i}, y_{i}\right) \pi_{i}\left(E_{i}\right) \geq \sum_{E_{i} \in \Pi_{i}} v_{i}\left(E_{i}, x_{i}\right) \pi_{i}\left(E_{i}\right)=V_{i}\left(x_{i}\right)$ for all $i \notin C$.

Since $\epsilon y_{i} \rightarrow y_{i}$ as $\epsilon \rightarrow 1$ in $\left(\mathbb{R}_{+}^{l}\right)^{\infty}$ and $V_{i}$ is continuous, there exists $\epsilon \in(0,1)$ such that

$$
V_{i}\left(\epsilon y_{i}\right)>V_{i}\left(x_{i}\right) \text { for all } i \in C \text {. }
$$

For all $\omega \in \Omega$, define

$$
z_{i}(\omega)= \begin{cases}\epsilon y_{i}(\omega) & \text { if } i \in C \\ y_{i}(\omega)+\frac{1-\epsilon}{\|I-C\|} \sum_{i \in C} y_{i}(\omega) & \text { if } i \notin C .\end{cases}
$$

Then $V_{i}\left(z_{i}\right)=V_{i}\left(\epsilon y_{i}\right)>V_{i}\left(x_{i}\right)$ for all $i \in C$. Moreover, since $u_{i}(\omega, \cdot)$ is strongly monotone, $V_{i}\left(z_{i}\right)=V_{i}\left(y_{i}+\frac{1-\epsilon}{\|I-C\|} \sum_{i \in C} y_{i}\right)>V_{i}\left(y_{i}\right) \geq V_{i}\left(x_{i}\right)$ for all $i \notin C$.

Then we check the feasibility of $z$, for every $\omega \in \Omega$,

$$
\begin{aligned}
\sum_{i \in I} z_{i}(\omega) & =\sum_{i \in C} \epsilon y_{i}(\omega)+\sum_{i \notin C} y_{i}(\omega)+(1-\epsilon) \sum_{i \in C} y_{i}(\omega) \\
& =\sum_{i \in I} y_{i}(\omega)=\sum_{i \in I} e_{i}(\omega) .
\end{aligned}
$$

Thus $\left\{x_{i}\right\}_{i \in I}$ is not maximin efficient, a contradiction.

\section{References}

A. Araujo, R. Novinski and M. R. Páscoa, General equilibrium, wariness and efficient bubbles, J. Econ. Theory 146 (2011), 785-811.

L. Angeloni and V. F. Martins-da-Rocha, Large economies with differential information and without free disposal, Econ. Theory 38 (2009), 263-286.

J. P. Aubin, Mathematical Methods of Game and Economic Theory, NorthHolland, Amsterdam, 1979.

R. J. Aumann, Markets with a continuum of traders, Econometrica 32 (1964), $39-50$

T. F. Bewley, Existence of equilibria in economies with infinitely many commodities, J. Econ. Theory 4 (1972), 514-540. 
A. Bhowmik and J. Cao, Blocking efficiency in an economy with asymmetric information, J. Math. Econ. 48 (2012a), 396-403.

A. Bhowmik and J. Cao, On the core and Walrasian expectations equilibrium in infinite dimensional commodity spaces, Econ. Theory (2012b), forthcoming.

A. Bhowmik and J. Cao, Robust efficiency in mixed economies with asymmetric information, J. Math. Econ. 49 (2013), 49-57.

J. Correia-da-Silva and C. Hervés-Beloso, Prudent expectations equilibrium in economies with uncertain delivery, Econ.Theory 39 (2009), 67-92.

L. I. de Castro, M. Pesce and N. C. Yannelis, Core and equilibria under ambiguity, Econ. Theory 48 (2011), 519-548.

L. I. de Castro, M. Pesce and N. C. Yannelis, A new perspective to rational expectations, working paper (2012).

L. I. de Castro and N. C. Yannelis, Ambiguity aversion solves the conflict between efficiency and incentive compatibility, working paper (2010).

G. Debreu and H. Scarf, A limit theorem on the core of an economy, Int. Econ. Rev. 4 (1963), 235-246.

E. Einy, D. Moreno and B. Shitovitz, Competitive and core allocations in large economies with differentiated information, Econ. Theory 18 (2001), 321-332.

E. Einy and B. Shitovitz, Private value allocations in large economies with differential information, Games Econ. Behav. 34 (2001), 287-311.

D. Ellsberg, Risk, ambiguity, and the savage axioms, Q. J. Econ. 75 (1961), 643669.

J. García-Cutrín and C. Hervés-Beloso, A discrete approach to continuum economies, Econ. Theory 3 (1993), 577-583.

I. Gilboa and D. Schmeidler, Maximin expected utility with non-unique prior, $J$. Math. Econ. 18 (1989), 141-153.

D. Glycopantis, A. Muir and N. C. Yannelis, On extensive form implementation of contracts in differential information economies, Econ. Theory 21 (2003), 495526.

D. Glycopantis and N. C. Yannelis, Differential information economies, Springer, 2005. 
M. G. Graziano and C. Meo, The Aubin private core of differential information economies, Decis. Econ. Finance 28 (2005), 9-31.

B. Grodal, A second remark on the core of an atomless economy, Econometrica 40 (1972), 581-583.

C. Hervés-Beloso and E. Moreno-García, Competitive equilibria and the grand coalition, J. Math. Econ. 44 (2008), 697-706.

C. Hervés-Beloso, E. Moreno-García and N. C. Yannelis, An equivalence theorem for differential information economy, J. Math. Econ. 41 (2005a), 844-856.

C. Hervés-Beloso, E. Moreno-García and N. C. Yannelis, Characterization and incentive compatibility of Walrasian expectations equilibrium in infinite dimensional commodity spaces, Econ. Theory 26 (2005b), 361-381.

W. Hildenbrand, Core and Equilibria of a Large Economy, Princeton Univ. Press, Princeton, NJ, 1974.

B. Holmstrom and R. B. Myerson, Efficient and durable decision rules with incomplete information, Econometrica 51 (1983), 1799-1819.

S. Krasa and N. C. Yannelis, The value allocation of an economy with differential information, Econometrica 62 (1994), 881-900.

L. C. Koutsougeras and N. C. Yannelis, Incentive compatibility and information superiority of the core of an economy with differential information, Econ. Theory 3 (1993), 195-216.

Z. Liu and N. C. Yannelis, Implementation under ambiguity: the maximin core, working paper (2013).

V. M. Marakulin, Equilibria and core as an essence of economic contractual interactions: mathematical foundations, Novosibirsk, 320 p., monograph under work, 2013.

K. Podczeck, R. Tourky and N. C. Yannelis, Non-existence of Radner equilibrium already with countably infinitely many states, University of Vienna, working paper (2008).

K. Podczeck and N. C. Yannelis, Equilibrium theory with asymmetric information and with infinitely many commodities. J. Econ. Theory 141 (2008), 152-183.

R. Radner, Competitive equilibrium under uncertainty, Econometrica 36 (1968), $31-58$. 
R. Radner, Equilibrium under uncertainty, in Handbook of mathematical economics, vol. II. (K. J. Arrow and M. D. Intriligator eds.) North Holland, Amsterdam, 1982.

D. Schmeidler, A remark on the core of an atomless economy, Econometrica $\mathbf{4 0}$ (1972), 579-580.

Y. Sun and N. C. Yannelis, Perfect competition in asymmetric information economies: compatibility of efficiency and incentives, J. Econ. Theory 134 (2007a), 175-194.

Y. Sun and N. C. Yannelis, Core, equilibria and incentives in large asymmetric information economies, Games Econ. Behav. 61 (2007b), 131-155.

Y. Sun and N. C. Yannelis, Ex ante efficiency implies incentive compatibility, Econ. Theory 36 (2008), 35-55.

K. Vind, A third remark on the core of an atomless economy, Econometrica 40 (1972), 585-586.

N. C. Yannelis, The core of an economy with differential information, Econ. Theory 1 (1991), 183-196. 